CALT-68-2065

hep-th/9607201

\title{
Lectures on Superstring and M Theory Dualitiest
}

\author{
John H. Schwarz \\ California Institute of Technology, Pasadena, CA 91125, USA
}

\begin{abstract}
These lectures begin by reviewing the evidence for $\mathrm{S}$ duality of the toroidally compactified heterotic string in 4d that was obtained in the period 1992-94. Next they review recently discovered dualities that relate all five of the $10 \mathrm{~d}$ superstring theories and a quantum extension of 11d supergravity called M theory. The study of $p$-branes of various dimensions (some of which are $D$-branes) plays a central role. The final sections survey supersymmetric string vacua in $6 \mathrm{~d}$ and some of the dual constructions by which they can be obtained. Special emphasis is given to a class of $N=1$ models that exhibit "heterotic-heterotic duality."
\end{abstract}

Lectures presented at the ICTP Spring School (March 1996)

and at the TASI Summer School (June 1996)

\footnotetext{
${ }^{1}$ Work supported in part by the U.S. Dept. of Energy under Grant No. DE-FG03-92-ER40701.
} 


\section{Introduction}

In the first superstring revolution (1984-85) we learned that there are just five superstring theories, each of which admits a 10d Poincaré-invariant vacuum and has a perturbation expansion that is consistent at every finite order. [1] Three of the theories have $N=1$ supersymmetry in 10d (type I and the two heterotic theories), one has $N=2$ supersymmetry in 10d with the two supercharges having opposite chirality (type IIA) and one has $N=2$ supersymmetry in 10d with the two supersymmetries having the same chirality (type IIB). One of the theories is based on unoriented open and closed strings (type I) and the other four are based on oriented closed strings. In short, each of the five theories appears to be quite different from the others, with very distinctive features. Of course, we don't really want five theories, since there is only one universe to explain. So the hope that I and others expressed in the mid 1980's was that some of these might turn out to be equivalent or inconsistent, but it wasn't apparent how this could happen.

In the second superstring revolution (1994-??) we are learning that all of these different superstring theories are consistent, but that they are non-perturbatively equivalent. Each of them represents a perturbation expansion of a single underlying theory about a distinct point in the moduli space of quantum vacua. Moreover, there is a sixth rather special point in this moduli space characterized by an 11d Poincaré-invariant vacuum. The rules for doing quantum mechanics in the $11 \mathrm{~d}$ vacuum are not yet understood, but the answer (whatever it is) has been named ' $M$ theory'. Some people believe that $M$ theory is more fundamental than the five superstring theories in 10d, but I do not share that viewpoint. Rather, for reasons that will be explained in these lectures, I believe that it is on a roughly equal footing with the type IIB superstring theory (or 'F theory'). Each of these descriptions (extended by various possible compactifications) gives access to different 'patches' of the space of quantum vacua. A good analogy, which I heard first from Vafa (at the CERN workshop in June 1996), is that the underlying theory is being defined in much the same way that one defines a manifold. A manifold can be defined by giving a covering by open sets that are diffeomorphic to open sets in $\mathbf{R}^{n}$ and by consistently defining transition functions on their overlaps. In the proposed analogy, each of the superstring theories corresponds to one of the open sets, and the dualities that characterize their non-perturbative equivalences correspond to the transition functions. 
From this viewpoint the various dualities could be viewed as part of the definition of the underlying theory rather than as conjectured theorems that require proof. Of course, as in the case of ordinary transition functions, they must satisfy a number of consistency conditions. Exactly what consistency conditions are required has not yet been carefully spelled out. There is a widespread belief that a deeper formulation of the theory ought to exist, and I tend to share that belief, but it is also conceivable that the various perturbation expansions and non-perturbative dualities constitute the best definition of the theory.

\subsection{Duality Symmetries of Supergravity and Superstring Theories}

There are three types of dualities that appear in superstring theory, which go by the names of $S, T$, and $U$. Two theories, call them $\mathrm{A}$ and $\mathrm{B}$, are said to be $S$ dual if theory $\mathrm{A}$ at strong coupling is equivalent to theory B at weak coupling (and vice versa). This means that there is an exact map between the A and B descriptions that includes, among other things the relation $\phi_{A}=-\phi_{B}$. Here $\phi_{A}$ and $\phi_{B}$ denote the respective dilaton fields, which determine the string coupling constant $\lambda$ according to the rule $\lambda=\exp \langle\phi\rangle$. Theories $\mathrm{A}$ and $\mathrm{B}$ are called $\mathrm{T}$ dual if theory $\mathrm{A}$ compactified on a space of large volume is equivalent to theory B compactified on a space of small volume (and vice versa). This means, for example, that some other scalar field $t$, the exponential of whose vev determines the volume of the compactified dimensions, satisfies $t_{A}=-t_{B}$. $T$ dualities can be checked order-by-order in string perturbation theory, and therefore they were the first ones to be understood. Theories A and $\mathrm{B}$ can be called $U$ dual if theory A compactified on a space of large (or small) volume is equivalent to theory $B$ at strong (or weak) coupling. In this case $t_{A}= \pm \phi_{B}$. This is not exactly the definition of $U$ duality that was originally proposed, but I feel it is in the spirit of the original proposal and find it to be convenient. When present, each of these dualities is supposed to constitute an exact quantum equivalence, which means that the two 'theories' should really be viewed as different descriptions of a single theory. It sometimes happens that a single theory is self-dual under a group of these dualities. In this case, the dualities are symmetries - discrete gauge symmetries, to be precise. This means that configurations related by duality transformations describe equivalent vacua, which should be identified as one and the same.

The appearance of a non-compact global symmetry group $G$ is a characteristic feature 
of the supergravity theories that represent the low-energy effective action for the massless modes of a superstring compactification. Typically, the group $G$ is realized nonlinearly by scalar fields that parametrize the homogeneous space $G / H$, where $H$ is the maximal compact subgroup of $G$. The first example of this phenomenon, with $G=S L(2, \mathbf{R})$ and $H=U(1)$, was uncovered in 1976 in a version of $N=44 \mathrm{~d}$ supergravity by Cremmer, Ferrara, and Scherk. 22] Curiously, a discrete subgroup of the symmetry of this particular example corresponds precisely to the example of $S$ duality that was first recognized in string theory - that of the toroidally compactified heterotic string. An analogous non-compact $E_{7}$ symmetry was found in $N=8$ 4d supergravity by Cremmer and Julia in 1978, [3] and many other examples were worked out thereafter. [1] The Cremmer-Julia example corresponds to the toroidally compactified type II string, and combines $S, T$, and $U$ dualities in a single discrete group. (As mentioned above, this usage of the term ' $U$ duality' differs a bit from the one proposed by Hull and Townsend, [5] which refers to the entire group as ' $U$ duality.')

The first proposal for the non-perturbative behavior of string theory was the 1990 suggestion of Font et al. [6] that the $S L(2, \mathbf{Z})$ subgroup of the $S L(2, \mathbf{R})$ of Cremmer, Ferrara, and Scherk should be an exact symmetry of the heterotic string toroidally compactified (in the way described by Narain[7]) to $4 \mathrm{~d}$. They named this discrete symmetry group $S$ duality, because the $N=1$ superfield (containing the axion and dilaton) that parametrizes $S L(2, \mathbf{R}) / U(1)$ is often called $S$. That $S$ duality should be an exact symmetry of the quantum string theory was a bold conjecture, since a $\mathbf{Z}_{2}$ subgroup is an electric-magnetic duality in which the coupling constant is inverted $\left(g_{e l} \rightarrow g_{m a g} \propto 1 / g_{e l}\right)$. Thus, it relates the strong coupling limit to a weakly coupled description. This proposal extends the duality conjecture of Montonen and Olive [11] from supersymmetric gauge theories to the superstring setting.

Since string theory had only been formulated in perturbation theory, the proposal of Font et al., when it first appeared, seemed to me to be an intriguing but untestable suggestion. In any case, the $S$ duality conjecture was eventually picked up and pursued by Sen and myself. [8, 9, 10] As we will see, non-trivial tests of $S$ duality have been formulated and verified. The technical tool that makes it possible to extract non-perturbative information about theories that have only been defined perturbatively is supersymmetry. Specifically, when there is enough supersymmetry, states belonging to 'short representations' of the supersymmetry algebra are exactly stable and have many of their properties protected from 
quantum corrections - both perturbative and non-perturbative. This will be discussed in more detail later.

$T$ duality, unlike $S$ duality, holds order by order in string perturbation theory. 112 In the simplest case - compactification on a circle - the group is $\mathbf{Z}_{2}$ and the transformation corresponds to inversion of the radius $\left(R \rightarrow \alpha^{\prime} / R\right)$. Of course, as mentioned above, $R$ is determined by the value of a scalar field (a $T$ modulus). As in the case of $S$ duality, when $T$ duality is a symmetry of a single theory, it is a discrete gauge symmetry that is realized as a field transformation, whereas when it relates two apparently different theories it is a field identification.

\subsection{The 4d Heterotic String}

In the example of toroidal compactification of the heterotic string no supersymmetry is broken, and in $4 \mathrm{~d}$ there are 132 scalar fields that live on the Narain moduli space $\mathcal{M}_{6,22}$. Narain spaces $\mathcal{M}_{k, l}$ are defined by

$$
\mathcal{M}_{k, l}=S O(k, l ; \mathbf{Z}) \backslash S O(k, l) / S O(k) \times S O(l) .
$$

It is convenient to introduce this notation here, since we shall encounter various Narain spaces in the course of these lectures. Recall that $S O(k, l)$ is the noncompact form of $S O(k+l)$ that preserves a metric with $k$ plus signs and $l$ minus signs. The group $S O(k) \times S O(l)$ is its maximal compact subgroup and the quotient space $S O(k, l) / S O(k) \times S O(l)$ is a homogeneous space of dimension $k l$. The discrete group $S O(k, l ; \mathbf{Z})$ is an infinite group consisting of all $S O(k, l)$ matrices with integer entries. When $l=k+16$, it is the subgroup of $S O(k, l)$ that preserves a certain even self-dual lattice of signature $(k, l)$ introduced by Narain. A homogeneous space is very smooth and well-behaved, but modding out by the discrete group introduces orbifold singularities, corresponding to the fixed points of the discrete group, in the moduli space. The $T$ duality group for the $4 \mathrm{~d}$ heterotic string is $G_{T}=S O(6,22 ; \mathbf{Z})$, and the 132 scalar fields belong to 22 Abelian $N=4$ gauge multiplets. In terms of compactification from 10d, 21 of the scalars originate from the metric, 15 from the two-form $B_{\mu \nu}$, and 96 from the $16 U(1)$ gauge fields that form the Cartan subalgebra of $E_{8} \times E_{8}$ or $S O(32)$.

The toroidally compactified heterotic string also contains two additional scalar fields called the axion $\chi$ and the dilaton $\phi$ - which belong to the $N=4$ supergravity multiplet. The 
dilaton is the $10 \mathrm{~d}$ dilaton shifted by a function of the other moduli such that the exponential of its vev gives the $4 \mathrm{~d}$ coupling constant. The $4 \mathrm{~d}$ axion is the scalar field that is dual to the two-form $B$ in $4 \mathrm{~d}$. The supergravity theory that contains these fields is precisely the one studied by Cremmer, Ferrara, and Scherk. They showed that $\chi$ and $\phi$ parametrize the homogeneous space $S L(2, \mathbf{R}) / U(1)$. Actually, in the quantum theory, only the discrete $S$ duality subgroup $S L(2, \mathbf{Z})$ is a symmetry, and the moduli space is

$$
\mathcal{M}_{S}=S L(2, \mathbf{Z}) \backslash S L(2, \mathbf{R}) / U(1)
$$

To see how this works, let us introduce a complex scalar field

$$
\rho=\chi+i e^{-2 \phi}=\rho_{1}+i \rho_{2}
$$

whose vev is $\left\langle\rho>=\theta / 2 \pi+i / \lambda^{2}\right.$, where $\theta$ is the vacuum angle and $\lambda$ is the coupling constant. $N=4$ Yang-Mills theories have vanishing $\beta$ function, so that $\theta$ and $\lambda$ are well-defined independent of scale. In terms of $\rho$, the $S L(2, \mathbf{Z})$ symmetry is realized by the non-linear transformations

$$
\rho \rightarrow \frac{a \rho+b}{c \rho+d}, \quad\left(\begin{array}{ll}
a & b \\
c & d
\end{array}\right) \in S L(2, \mathbf{Z}) .
$$

As usual, when instanton effects are taken into account, the continuous Peccei-Quinn symmetry $\chi \rightarrow \chi+b$, is broken to the discrete subgroup for which $b$ is an integer. This subgroup and the inversion $\rho \rightarrow-1 / \rho$ generate the discrete group $S L(2, \mathbf{Z})$ or, when matrices are not distinguished from their negatives, $\operatorname{PSL}(2, \mathbf{Z})$. When $\theta=0$, a special case is inversion of the coupling constant $\lambda \rightarrow 1 / \lambda$. In general, the $S L(2, \mathbf{Z})$ symmetry of the theory is broken completely by any specific choice of vacuum. Only when the vev of $\rho$ is at one of the orbifold points of the moduli space does some unbroken symmetry $\left(\mathbf{Z}_{2}\right.$ or $\left.\mathbf{Z}_{3}\right)$ remain.

Mathematically, $S$ and $T$ duality are quite analogous in the 4 d low-energy effective field theory, even though their implications for string theory are dramatically different. This analogy was one of the original motivations for proposing that $S$ duality should also be a symmetry. The massless bosonic fields of the toroidally compactified heterotic string are the metric tensor $g_{\mu \nu}$, the axion-dilaton field $\rho, 28$ Abelian gauge fields $A_{\mu}^{a}$ (6 from the 10d metric, 6 from the 10d two-form, and 16 from the Cartan subalgebra), and the 132 moduli parametrizing $\mathcal{M}_{6,22}$. These are the only massless bosonic fields at generic points in the 
classical moduli space. At the singular points, where there is enhanced gauge symmetry, there are more. The 132 moduli are conveniently described as a symmetric $28 \times 28$ matrix belonging to the group $S O(6,22)$ :

$$
\begin{aligned}
M^{T} & =M, \quad M^{T} L M=L \\
L & =\left(\begin{array}{ccc}
0 & I_{6} & 0 \\
I_{6} & 0 & 0 \\
0 & 0 & I_{22}
\end{array}\right) .
\end{aligned}
$$

Under a $T$ duality transformation given by an $S O(6,22 ; \mathbf{Z})$ matrix $\Omega$ satisfying $\Omega^{T} L \Omega=L$

$$
M \rightarrow \Omega M \Omega^{T}, \quad A_{\mu} \rightarrow \Omega A_{\mu},
$$

while $g_{\mu \nu}$ and $\rho$ are invariant.

The $28 \mathrm{U}(1)$ gauge fields $A_{\mu}^{a}$ give rise to 28 electric and 28 magnetic charges. A convenient way to define them is to assume that space-time is asymptotically flat and use the asymptotic behavior of the field strengths:

$$
F_{0 i}^{a} \sim \frac{q_{e l}^{a}}{r^{3}} x^{i} \quad \tilde{F}_{0 i}^{a} \sim \frac{q_{m a g}^{a}}{r^{3}} x^{i}
$$

The allowed charges are controlled by the asymptotic values of the moduli $\left(\rho \sim \rho^{(0)}\right.$ and $M_{a b} \sim M_{a b}^{(0)}$ ) and a pair of vectors $\alpha^{a}, \beta^{a}$ belonging to the Narain lattice, which is an even self-dual Lorentzian lattice of signature $(6,22)$. The formulas are

$$
q_{e l}^{a}=\frac{1}{\rho_{2}^{(0)}} M_{a b}^{(0)}\left(\alpha^{b}+\rho_{1}^{(0)} \beta^{b}\right), \quad q_{m a g}^{a}=L_{a b} \beta^{b} .
$$

These formulas automatically incorporate the Dirac-Schwinger-Zwanziger-Witten quantization rules (i.e., the quantization condition for dyons in the presence of a $\theta$ angle). States in the perturbative string spectrum carry electric charge only and therefore have $\beta^{a}=0$.

\subsection{The 4d Type II Superstring}

The type II (A or B) superstring compactified on $T^{6}$ is approximated at low-energy by $N=8$ supergravity. The classical theory has a non-compact symmetry group $E_{7,7}$. The natural conjecture for the duality group in this case is the discrete subgroup $E_{7}(\mathbf{Z})$, which is defined as the intersection of the continuous $E_{7,7}$ group and the discrete group $S p(28 ; \mathbf{Z})$. [5 Written 
in the 56-dimensional fundamental representation, it is evident that $E_{7,7}$ is a subgroup of the non-compact group $S p(28)$. (Later, in other contexts, the symbol $S p(n)$ will represent a compact group.) The analog of the Narain moduli space in this case is

$$
\mathcal{M}=E_{7}(\mathbf{Z}) \backslash E_{7,7} / S U(8)
$$

The scalar fields of $N=8$ supergravity parametrize this 70-dimensional space. As in the $N=4$ heterotic theory, there are once again $28 U(1)$ gauge fields. However, this time only 12 of their electric charges are excited (by Kaluza-Klein and winding excitations) in the perturbative string spectrum. The remaining 16 electric charges and all of the magnetic charges are only carried by non-perturbative excitations. One way of understanding this is to note the decomposition

$$
E_{7}(\mathbf{Z}) \supset S O(6,6 ; \mathbf{Z}) \times S L(2, \mathbf{Z}),
$$

which exhibits the $T$ duality and $S$ duality subgroups. With respect to this subgroup, the fundamental 56 representation decomposes as $\mathbf{5 6}=(\mathbf{1 2}, \mathbf{2})+(\mathbf{3 2}, \mathbf{1})$. The 12 electric charges that occur perturbatively are carried by states whose mass is finite at weak coupling in the string metric. They are associated to the first term, as are the dual magnetic charges, which give states whose mass is proportional to $1 / \lambda^{2}$. The 16 electric and magnetic charges associated to the 32-dimensional spinorial representation of $S O(6,6)$ turn out to be carried by $D$-branes, and as a result they give masses proportional to $1 / \lambda$.

\subsection{The BPS Condition}

The $N$-extended $4 \mathrm{~d}$ supersymmetry algebra (in 2-component notation) includes the anticommutator

$$
\left\{Q_{\alpha}^{I}, Q_{\beta}^{J}\right\}=\epsilon_{\alpha \beta} Z^{I J}
$$

The $N(N-1) / 2$ central charges $Z^{I J}=-Z^{J I}$ are complex numbers whose real and imaginary parts give the electric and magnetic charges associated with the $N(N-1) / 2 U(1)$ gauge fields in the $N$-extended 4 d supergravity multiplet. The supersymmetry algebra implies that the mass of any state is bounded below by its central charges. This bound, known as the Bogomol'nyi bound, is very important. When the mass of a state attains the minimum value 
allowed for given charges (and moduli), the state is said to be BPS saturated. BPS states belong to smaller representations of the algebra than are possible when the bound is not saturated. There are actually several possibilities for how this can be achieved. To explain this, it is convenient to make an $S O(N)$ change of basis such that (in the case of $N=4$, for example)

$$
Z=\left(\begin{array}{cccc}
0 & Z_{1} & 0 & 0 \\
-Z_{1} & 0 & 0 & 0 \\
0 & 0 & 0 & Z_{2} \\
0 & 0 & -Z_{2} & 0
\end{array}\right)
$$

Thus we see that in the $N=4$ case, even though the supergravity multiplet has six $U(1)$ gauge fields, a generic configuration can be described by only considering two electric and two magnetic charges. In this case there are two ways to achieve BPS saturation. In the first case, the mass satisfies the relations $M=\left|Z_{1}\right|=\left|Z_{2}\right|$. This gives 'ultrashort' multiplets, such as the 16-dimensional gauge multiplet. The second possibility for a BPS state is $M=\left|Z_{1}\right|>\left|Z_{2}\right|$. The first case occurs when the electric charge vector $\alpha^{a}$ and the magnetic charge vector $\beta^{a}$ are parallel, while in the second case they are not parallel. Since BPS states in the perturbative string spectrum are purely electric, they are necessarily of the first type.

These considerations are important in making comparisons of string states and black holes. Static extremal black hole configurations with $M=\left|Z_{1}\right|=\left|Z_{2}\right|$ turn out to preserve one-half of the supersymmetry and to have a horizon of vanishing area (and hence no Bekenstein-Hawking entropy). Ones with $M=\left|Z_{1}\right|>\left|Z_{2}\right|$, on the other hand, preserve only one-quarter of the supersymmetry and have a horizon of finite area. There are analogous statements that can be made in the $N=8$ case. In that case, in order to obtain a finite-area horizon, it is necessary that $M$ equals only one of the four $\left|Z_{i}\right|$ 's so that seven-eighths of the supersymmetry is broken. There has been dramatic progress recently in accounting for the entropy of supersymmetric black holes with finite area horizons in terms of the counting of microscopic string degrees of freedom. However, I will leave that (and generalizations) to other lecturers. 


\subsection{Tests of S Duality}

$T$ duality works perturbatively and is well understood, but how can we prove $S$ duality without knowing non-perturbative string theory? As yet, we cannot prove it, but we can subject the conjecture to some non-trivial tests by focusing on BPS states. The essential fact, pointed out long ago by Witten and Olive, 13 is that such states can receive no quantum corrections - perturbative or nonperturbative - to their masses so long as the supersymmetry remains unbroken. Thus, a non-trivial prediction of $S$ duality, which we can attempt to check, is that the multiplicities of BPS states are $S L(2, \mathbf{Z})$ invariant. Note that since the vacuum breaks $S$ and $T$ duality spontaneously, the BPS states do not form degenerate multiplets.

Let us now explore which states in the elementary string spectrum of the $4 \mathrm{~d}$ heterotic string saturate the Bogomol'nyi bound. Absorbing the moduli $M^{(0)}$ in the definition of the Narain lattice, the BPS condition for purely electric states becomes

$$
(\mathrm{Mass})^{2}=\frac{1}{16 \rho_{2}^{(0)}} \hat{\alpha}^{a}(I+L)_{a b} \hat{\alpha}^{b}=\frac{1}{8 \rho_{2}^{(0)}}\left(\hat{\alpha}_{R}\right)^{2},
$$

where $\hat{\alpha} L \hat{\alpha}=\hat{\alpha}_{R} \cdot \hat{\alpha}_{R}-\hat{\alpha}_{L} \cdot \hat{\alpha}_{L}$. ( $\hat{\alpha}_{L}$ is 22-dimensional and $\hat{\alpha}_{R}$ is 6-dimensional. They correspond to the left-moving and right-moving internal momenta of the string.) Now we should compare the free string spectrum, which is given by

$$
(\mathrm{Mass})^{2}=\frac{1}{4 \rho_{2}^{(0)}}\left[\frac{1}{2}\left(\hat{\alpha}_{L}\right)^{2}+N_{L}-1\right]=\frac{1}{4 \rho_{2}^{(0)}}\left[\frac{1}{2}\left(\hat{\alpha}_{R}\right)^{2}+N_{R}-\delta\right] .
$$

$N_{L}$ and $N_{R}$ represent left-moving and right-moving oscillator excitations. The parameter $\delta$ is $1 / 2$ in the NS sector and 0 in the $\mathrm{R}$ sector. Alternatively, it is simply 0 in the GS formulation. The factor of $\left(\rho_{2}^{(0)}\right)^{-1}$ appears because the mass is computed with respect to the canonically normalized Einstein metric. It does not appear if one uses the string metric, which differs by a dilaton-dependent Weyl rescaling. The Einstein metric is more natural in the present context, because it is invariant under $S$ duality transformations. Comparing formulas, one sees that the Bogomol'nyi bound is saturated provided that $N_{R}=\delta$ (which gives eight bosonic and eight fermionic right-moving modes - the short representation of $N=4)$ and $N_{L}=1+\frac{1}{2} \hat{\alpha} L \hat{\alpha}$. Thus, if $\hat{\alpha} L \hat{\alpha}=2 n-2$, for a non-negative integer $n$, then there is a short $N=4$ multiplet for every solution of $N_{L}=n$. These states are only "electrically" charged. The challenge is to find their predicted $S$ duality partners. Specifically, every 
elementary string excitation of the type we have just described $(\vec{\alpha}=\vec{\ell}, \vec{\beta}=0)$ should have magnetically charged partners with $\vec{\alpha}=a \vec{\ell}$ and $\vec{\beta}=c \vec{\ell}$. Since $a$ and $c$ are elements of an $S L(2, \mathbf{Z})$ matrix, they are relatively prime integers.

Sen has investigated the partners of electrically charged states with $\hat{\alpha} L \hat{\alpha}=-2$ (i.e., $N_{L}=0$ ). [14] He has shown that $S$ duality partners with $c=1$ can be identified with BPS monopole solutions (and their dyonic generalizations) of the effective field theory. These solutions saturate the bound, of course. Thus, as we have explained, they should persist with exactly this mass in the complete quantum string theory. For $c>1$, Sen argued that one should examine multi-BPS dyon bound states. Specifically, he showed that the prediction of $S$ duality is that each multi-BPS dyon moduli space should admit a unique normalizable harmonic form. Poincaré duality would give a second one unless it is self-dual or anti-selfdual. He constructed such an anti-self-dual form explicitly for the case of $c=2$, 15 providing the first really non-trivial test of $S$ duality. Progress toward extending this result to $c>2$ has been made by Segal and Selby [16] and by Porrati. [17] More recently, a simpler and more general proof has been constructed [18] using D-brane techniques. [19].

\section{DUALITIES IN NINE DIMENSIONS}

\section{$2.1 \quad$ Introductory Comments}

The five $10 \mathrm{~d}$ superstring theories - types I, IIA, IIB and the $E_{8} \times E_{8}$ and $S O(32)$ heterotic - are related to one another by a rich variety of dualities. The dualities that require compactification of only one spatial dimension, leaving a 9d Minkowski space-time, are sufficient to show that all five are related to one another. This strongly suggests that they are best regarded as different descriptions of a single underlying theory. Each one is better suited to describing some portion of the moduli space of possible vacua than the others. In a later section, we will discuss some of the additional dualities that emerge upon compactification to $6 \mathrm{~d}$, but in this section we wish to explore what can be learned while retaining an uncompactified $\mathbf{R}^{9}$.

Two of the relevant dualities are $T$ dualities, which can be understood perturbatively, and therefore they were understood prior to the recent non-perturbative discoveries. When the IIA and IIB theories are each compactified on a circle, so that altogether the space-time 
topology is $\mathbf{R}^{9} \times S^{1}$, the two theories are $T$ dual. 20, 21] This means that they describe identical physics, provided that the radius of one circle is the inverse of the other one (in string units). In a similar manner, one can show that the $S O(32)$ and $E_{8} \times E_{8}$ heterotic string theories are $T$ dual when each of them is compactified on a circle. [0, 22] This heterotic case is somewhat more subtle than the type II one. Wilson lines have to be included, as part of the characterization of the compactification, in order to match corresponding points in the moduli space of $9 \mathrm{~d}$ vacua. The relevant moduli space in this case is the Narain space $\mathcal{M}_{1,17}$ defined earlier.

The pair of $T$ dualities described above provides two connections among the five superstring theories. To see that all five are connected requires examining non-perturbative dualities - analogs of the $S$ duality of the $4 \mathrm{~d}$ heterotic string discussed in Section 1. One way of addressing the problem is to ask, for each of the five theories, whether the strong coupling limit has a dual weakly coupled description. As in the case of the $S$ duality of the $4 \mathrm{~d}$ heterotic string, the procedure is to identify a plausible candidate for an $S$ dual description and then to examine its consequences. The result is that an interesting and consistent story emerges. In fact, it is so compelling that there can be little doubt about the truth of the proposed dualities. So let me now say what they are.

The type I and $S O(32)$ heterotic string theories are $S$ dual. 23, 24] This means that the respective dilatons are related by $\phi_{I}=-\phi_{H}$, so that the coupling constants $\left(\lambda=e^{<\phi>}\right)$ are reciprocal to one another. This identification is supported by the fact that both have the same low-energy effective field theory descriptions $(N=1$ supergravity coupled to $S O(32)$ super Yang-Mills in 10d). The field redefinition $\phi_{I}=-\phi_{H}$ must be accompanied by a Weyl rescaling of the metric to convert from one version of the action to the other. On the other hand, the type IIB superstring in 10d is self-dual. [5] More precisely, there is an $S L(2, \mathbf{Z})$ $S$-duality group, very much like that of the $4 \mathrm{~d}$ heterotic string, that is a gauge symmetry of the theory. This will be described in detail later.

The strong coupling limits of the IIA and $E_{8} \times E_{8}$ theories turn out to provide quite a different surprise. In each case there is an eleventh dimension (tenth spatial dimension) that becomes large at strong coupling. 25, 23, 26] Specifically, the size of this dimension scales as $L_{11} \sim \lambda^{2 / 3}$, where $\lambda$ is the $10 \mathrm{~d}$ coupling constant. Such a compact dimension is completely invisible in perturbation theory (an expansion about $\lambda=0$ ), which is why it 
passed unnoticed for so many years. In the IIA case, the hidden dimension is a circle $S^{1}$, whereas in the $E_{8} \times E_{8}$ case it is a line interval $I$, or (more precisely) an $S^{1} / \mathbf{Z}_{2}$ orbifold. This means that in the $E_{8} \times E_{8}$ case one can visualize the space-time as an $11 \mathrm{~d}$ space-time with two 10d faces, which are sometimes referred to as "end-of-the-world 9-branes," since they have nine spatial dimensions. [26] One of the $E_{8}$ gauge groups is associated to each face. In any case, at strong coupling the faces move apart and (away from the faces) the theory is described by the same 11d bulk theory that describes the IIA theory at strong coupling. This 11d theory is described in leading order in a low-energy expansion by 11d supergravity, a classical field theory that was discovered almost 20 years ago. 27 It is not yet known what is the correct algorithm that determines all the higher-dimension terms of the low-energy expansion of the effective action, but since we are confident that there is a consistent quantum theory, such an algorithm should exist. The unknown 11d quantum theory is referred to as $\mathrm{M}$ theory. As we will discuss, certain of its supersymmetric solitons are known, and they provide a handle on many of its interesting properties.

The equivalences discussed above can be summarized by the diagram in Figure 1. This diagram is sufficient to show that the five superstring theories are all part of a single structure, but it is by no means the whole story. There are a variety of other surprising dualities that are only revealed upon compactification of additional dimensions. Some of these will be described later.

The IIA/IIB $T$ duality and the IIA/M $S$ duality can be combined as a duality between IIB theory on $\mathbf{R}^{9} \times S^{1}$ and $\mathrm{M}$ theory on $\mathbf{R}^{9} \times T^{2}$. This viewpoint turns out to be very powerful for understanding the structure of both the IIB and M theories separately, as will be discussed in considerable detail. The $T^{2}$ is characterized by three real parameters - its area $A_{M}$ and its modular parameter $\tau$, which characterizes its complex structure up to an $S L(2, \mathbf{Z})$ transformation. Indeed, we will find that in $9 \mathrm{~d}$ the $S L(2, \mathbf{Z})$ modular group of the torus precisely corresponds to the $S L(2, \mathbf{Z}) S$ duality group of the IIB theory. This geometrization of $S$ duality is quite profound.

There is an analogous duality relating $\mathrm{M}$ theory and the $S O(32)$ theory (both type I and heterotic). This duality can be understood as arising as a corollary of the first one after modding out by a suitable $\mathbf{Z}_{2}$ symmetry. In this case, $\mathrm{M}$ theory compactified on a cylinder $C=I \times S^{1}$ is dual to the $S O(32)$ theory compactified on a circle $S^{1}$. This also has 


\section{$\mathrm{D}=\mathbf{1 1}$}

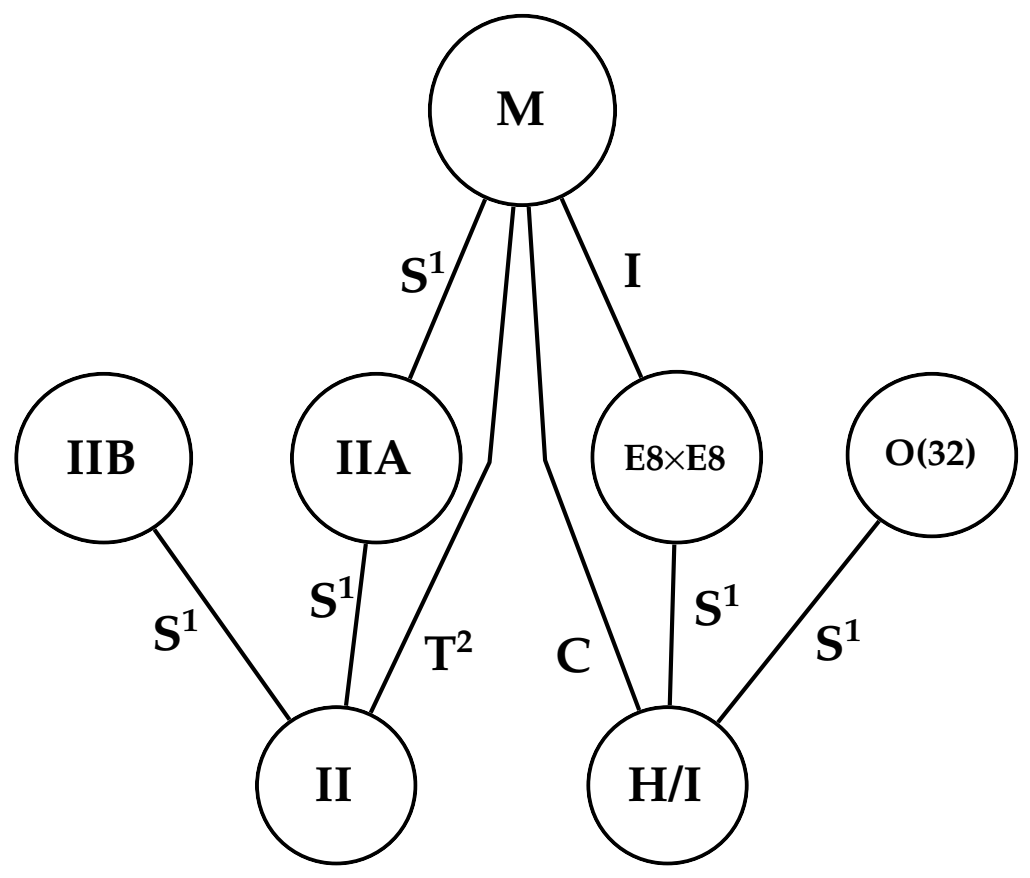

Figure 1: Duality Connections.

consequences for both theories.

\subsection{General Features of $p$-branes}

$M$ theory and the various superstring theories admit a rich variety of soliton solutions. When the core of such a solution extends over $p$ spatial dimensions (and one time dimension), the soliton is called a $p$-brane. Of special interest are $p$-brane solitons that saturate a BPS bound, which means that they preserve some fraction of the underlying supersymmetry. We will focus on ones that preserve one-half of the supersymmetry, but ones that preserve a smaller fraction, such as one-quarter or one-eighth, can be constructed. 
Supersymmetric $p$-branes are a natural generalization of the BPS states (0-branes) discussed in Section 1. In this case the relevant central charges in the supersymmetry algebra are $p$-forms. 28] Their magnitudes give a lower bound on the $p$-brane tension $T_{p}$, which is the mass per unit volume of the brane. Supersymmetry is preserved when the bound is saturated. As in the case of the 0-branes, the BPS condition ensures that solutions of classical low-energy supergravity field equations exhibit some features of the exact quantum string theory, such as the relationship between the tension and the charge.

The supergravity solutions are non-singular in certain cases, so that the energy is smoothly spread over a region surrounding a $p$-dimensional subspace. In other cases there are delta function singularities at the core that can be compensated by postulating the presence of "fundamental p-branes." Many authors distinguish these two categories of $p$-branes by calling them "solitonic" and "fundamental," respectively. As far as I can tell, this is a distinction that need not persist in the underlying quantum theory, rather it could just be an artifact of formalism and approximations. Therefore, we regard both categories of $p$-brane solutions as "solitonic" without focussing on this distinction. (See Ref. [29] for a review of $p$-brane solutions.)

The effective supergravities in question contain various antisymmetric tensor gauge fields. These can be represented as differential forms

$$
A_{n}=A_{\mu_{1} \mu_{2} \ldots \mu_{n}} d x^{\mu_{1}} \wedge d x^{\mu_{2}} \wedge \ldots \wedge d x^{\mu_{n}}
$$

In this notation, a gauge transformation is given by $\delta A_{n}=d \Lambda_{n-1}$, and the gauge-invariant field strength is $F_{n+1}=d A_{n}$. When interactions are included, these formulas are sometimes modified. The origin of $p$-branes can be understood by considering an action that (schematically) has the structure 30]

$$
S \sim \int d^{D} x \sqrt{-g}\left\{R+(\partial \phi)^{2}+e^{-a \phi} F_{n+1}^{2}+\ldots\right\}
$$

Here, $\phi$ represents a dilaton field, $R$ is the scalar curvature, and $a$ is a numerical constant whose value depends on the particular theory. The dots include all the additional terms required to make the theory locally supersymmetric. In this case it is meaningful to seek BPS $p$-brane solutions, and it turns out that solutions exist for $p=n-1$ and $p=D-n-3$. By a straightforward generalization of the nomenclature of Maxwell theory, it is natural to 
call these "electric" and "magnetic," respectively. The electric $p$-brane, with $p=n-1$, has an $n$-dimensional world-volume. The fact that it is a source for "electric" charge is exhibited by the coupling

$$
\int A_{\mu_{1} \ldots \mu_{n}} \frac{\partial x^{\mu_{1}}}{\partial \sigma_{1}} \ldots \frac{\partial x^{\mu_{n}}}{\partial \sigma_{n}} d^{n} \sigma
$$

which generalizes the familiar $j \cdot A$ coupling of Maxwell theory.

A $p$-brane in $D$ dimensions (let's assume it is an infinite hyperplane, for simplicity) can be encircled by a $(D-p-2)$-dimensional sphere $S^{D-p-2}$. Thus, the "electric charge" of the $p$-brane is given by a straightforward generalization of Gauss's law for point charges

$$
Q_{E} \sim \int_{S^{D-p-2}} * F
$$

where $* F$ is the Hodge dual of $F$. In these lectures, we will not need to commit ourselves to specific normalization conventions. Similarly, a dual $(D-p-4)$-brane has "magnetic charge"

$$
Q_{M} \sim \int_{S^{p+2}} F
$$

Note that the charge associated with a $p$-brane has dimension $(\text { length })^{D / 2-2-p}$. This is dimensionless when $p=(D-4) / 2-i . e$., for point particles in $4 \mathrm{~d}$, strings in $6 \mathrm{~d}$, membranes in $8 \mathrm{~d}$, etc. In these cases the electric and magnetic branes have the same dimensionality and it is possible to have dyonic $p$-branes.

The charges of $p$-branes can also be described by generalizations of Coulomb's law. So, for an electric $p$-brane, as $r \rightarrow \infty$

$$
A \sim \frac{Q_{E}}{r^{D-p-3}} \omega_{p+1}
$$

where $r$ is the transverse distance from the brane and $\omega_{p+1}$ is the volume form for the $p$-brane world-volume. Similarly, for the dual magnetic $(D-p-4)$-brane, as $r \rightarrow \infty$

$$
F \sim \frac{Q_{M}}{r^{p+2}} \Omega_{p+2}
$$

where $\Omega_{p+2}$ is the volume form on a sphere $S^{p+2}$ surrounding the brane. In this case it is convenient to describe the magnetic field, rather than the potential, in order to avoid introducing generalizations of Dirac strings. Of course, the distinction between electric and magnetic branes is not so great, since it is often possible to make a duality transformation 
that replaces $A$ by a dual potential $\tilde{A}$ whose field strength $d \tilde{A}$ is the dual of $F=d A$. From the

point of view of $\tilde{A}$, the original electric brane is magnetic and vice versa. Another significant fact,[31] noted more than ten years ago, is that the Dirac quantization condition has a straightforward generalization to the charges carried by a dual pair of $p$-branes: $Q_{E} Q_{M} \in$ $2 \pi \mathbf{Z}$. This assumes appropriate normalization conventions, of course.

The crudest first approximation to classical p-brane dynamics is given by a straightforward generalization of the Nambu area formula for the string world-sheet action. This gives an action proportional to the $(p+1)$-dimensional volume induced by embedding the world volume into the $D$-dimensional target space:

$$
S_{\text {eff }}=T_{P} \int \sqrt{\operatorname{det} G_{\alpha \beta}} d^{p+1} \sigma,
$$

where

$$
G_{\alpha \beta}=\eta_{\mu \nu} \partial_{\alpha} x^{\mu} \partial_{\beta} x^{\nu}, \alpha, \beta=0,1, \ldots, p,
$$

and $\eta$ is the metric (Minkowski, for example) of the target space. Just as for strings, this formula is invariant under reparametrizations of the world volume. Also, it defines the $p$-brane tension $T_{p}$ - the universal mass per unit volume of the $p$-brane. Note that $T_{p} \sim(\text { mass })^{p+1}$.

\section{$2.3 \quad$ Specific $p$-branes}

Let us now examine what BPS $p$-branes occur in the theories of most interest to us. We begin with 11d supergravity, the low-energy effective field theory for M theory. 11d supergravity has three massless fields: the metric $g_{\mu \nu}$ (with 44 physical polarizations), the gravitino $\psi_{\mu}$ (with 128 physical polarizations), and a three-form potential $C_{\mu \nu \rho}$ (with 84 physical polarizations). By the reasoning given above one expects to find two kinds of branes associated with $C$ : an electric 2-brane [45, 32] and a magnetic 5-brane, 33] and this is indeed the case, even though 11d supergravity has no dilaton field. These branes have a number of interesting properties, which we will return to later.

The only anomaly-free $10 \mathrm{~d}$ theories with $N=1$ supersymmetry have as their massless sector $N=1$ supergravity coupling to either $S O(32)$ or $E_{8} \times E_{8}$ super Yang-Mills matter. 34] The relevant antisymmetric tensor gauge field that couples to $p$-branes is the two-form potential $B$ belonging to the supergravity multiplet. In this case the electric $p$-brane is a 1-brane, 
which is the heterotic string. [35] Its magnetic dual is a 5-brane. [36] Type I strings cannot be found in this way, because they are not BPS (and hence not stable).

Type IIA supergravity in 10d can be understood as arising from compactification of M theory on a circle. 25, 23] Doing this, the $11 \mathrm{~d}$ metric gives rise to the $10 \mathrm{~d}$ metric, a one-form $A$, and a dilaton $\phi_{A}$. Specifically, if $g^{(10)}$ denotes the IIA string metric,

$$
g_{M N}^{(11)} d x^{M} d x^{N}=e^{-2 \phi_{A} / 3} g_{\mu \nu}^{(10)} d x^{\mu} d x^{\nu}+e^{4 \phi_{A} / 3}\left(d x^{11}-A_{\mu} d x^{\mu}\right)^{2}
$$

Identifying the string coupling constant $\lambda=e^{<\phi_{A}>}$, one sees that $R_{11} \sim \lambda^{2 / 3}$, as was asserted earlier. Also, the 11d three-form $C$ decomposes in 10d into a three-form $C$ and a two-form $B$.

The IIA theory has six kinds of $p$-branes: $p=0,6$ associated to $A ; p=1,5$ associated to $B ; p=2,4$ associated to $C$. Some of these have a simple interpretation in terms of the 2-brane and 5-brane in $11 \mathrm{~d}$. The 2-brane and 5-brane in $10 \mathrm{~d}$ are given by a straight dimensional reduction and the 1-brane and 4-brane in 10d are given by a double dimensional reduction. The fact that a $p$-brane solution in $D$ dimensions implies that there is also one in $D-1$ dimensions (for $p<D-3$ ) after compactification on a circle depends crucially on the BPS property. This allows one to form an infinite periodic array of parallel $p$-branes in $D$ dimensions, and then a periodic identification gives a single $p$-brane in $D-1$ dimensions. Double dimensional reduction is more straightforward: one dimension of the $p$-brane wraps around the circular dimension of the space-time. The 0-brane and 6-brane couple to the Kaluza-Klein gauge field $A$, and can therefore be called Kaluza-Klein $p$-branes. The charge carried by a KK 0-brane is interpreted as momentum in the 11th dimension. Their role in $11 \mathrm{~d}$ is simply to allow this momentum to be excited. The dual 6-brane, on the other hand, has a tension that diverges in the decompactification limit. Thus, there is no corresponding soliton in 11d Minkowski space.

Let us now consider the most interesting case of all - type IIB superstrings in 10d. 38 In the NS-NS sector there is a 2 -form potential $B^{(1)}$. The fundamental IIB string is electrically charged with respect to this field. In addition, the R-R sector has a zero-form $\chi$, a two-form $B^{(2)}$, and a four-form $A_{4}$. The four-form $A_{4}$ has a self-dual field strength $\left(d A_{4}=* d A_{4}\right)$, something that is possible only when the number of spatial dimensions minus the number of

\footnotetext{
${ }^{2}$ Another possibility, 8-branes, will not be considered here. (See Ref. [37].)
} 
time dimensions is a multiple of four.

Formally, a zero-form gives a (-1)-brane and a 7-brane, both of which are rather special. A (-1)-brane has a point-like world volume. After a Wick rotation, it can be interpreted as a kind of instanton called a D-Instanton. Its magnetic dual, a 7-brane, is also special. Whenever $p=D-3$, the presence of the brane gives rise to a conical deficit angle in the geometry of the transverse plane, a fact that is artfully exploited by $\mathrm{F}$ theory. [39] Here, we will only consider branes with $p<D-3$. The four-form $A_{4}$ gives rise to a self-dual 3-brane. It has an identified electric and magnetic charge, because $\int_{S^{5}} F_{5}=\int_{S^{5}} * F_{5}$. Thus, $A_{4}$ only gives one kind of $p$-brane. Finally, we turn to the two forms $B_{\mu \nu}^{(1)}$ and $B_{\mu \nu}^{(2)}$. Each can couple to an electric 1-brane or a magnetic 5-brane. However, as we will argue, these 1-branes or 5-branes can form bound states. Thus, we will get an infinite family of strings labelled by two electric charges $\left(q_{1}, q_{2}\right)$ and an infinite family dual magnetic 5-branes labelled by two magnetic charges.

\subsection{Type IIB String Solitons}

As has already been noted, the type IIB superstring in 10d has two two-form potentials, $B_{\mu \nu}^{(1)}$ and $B_{\mu \nu}^{(2)}$. Therefore, string-like solutions can, in general, carry a pair of charges

$$
q_{I} \sim \int_{S^{7}} * d B^{(I)}, \quad I=1,2
$$

Let us construct these solutions explicitly. To make the $S L(2, \mathbf{R})$ symmetry of the supergravity field equations manifest, it is convenient to introduce a two-component vector notation

$$
H=d B=\left(\begin{array}{l}
d B^{(1)} \\
d B^{(2)}
\end{array}\right) .
$$

It is also convenient to combine the $R R$ scalar $\chi$ and the dilaton $\phi$ into a complex scalar field

$$
\rho=\chi+i e^{-\phi}
$$

and to represent the vev of this field by

$$
<\rho>=\rho_{0}=\chi_{0}+i e^{-\phi_{0}}=\frac{\theta}{2 \pi}+\frac{i}{\lambda_{B}} .
$$


The field $\rho$ is very similar to the axion-dilaton field of the $4 \mathrm{~d}$ heterotic theory described in Section 1. Indeed, as in that case, it transforms nonlinearly under an $S L(2, \mathbf{R})$ transformation $\Lambda=\left(\begin{array}{ll}a & b \\ c & d\end{array}\right)$, by the rule $\rho \rightarrow \frac{a \rho+b}{c \rho+d}$. There are differences of detail, however. For example, the imaginary part of $\rho$ is $e^{-\phi}$ here, whereas in Section 1 it was $e^{-2 \phi}$. It is also convenient to introduce the symmetric $S L(2, \mathbf{R})$ matrix

$$
\mathcal{M}=e^{\phi}\left(\begin{array}{cc}
|\rho|^{2} & \chi \\
\chi & 1
\end{array}\right),
$$

which transforms by the simple rule

$$
\mathcal{M} \rightarrow \Lambda \mathcal{M} \Lambda^{T}
$$

The $B$ fields transform linearly by the rule $B \rightarrow\left(\Lambda^{T}\right)^{-1} B$, while the canonical metric $g_{\mu \nu}$ and the four-form $A_{4}$ are invariant. Note that since the dilaton transforms, the IIB string metric

$$
g_{\mu \nu}^{(B)}=e^{\phi / 2} g_{\mu \nu}
$$

is not $S L(2, \mathbf{R})$ invariant. For this reason, it is convenient to use the canonical metric for the time being.

The string-like solutions of the IIB supergravity field equations that we are seeking have $A_{4}$ and all fermi fields equal to zero. While it is difficult to formulate a convenient action that gives the complete field equations (because the field strength of $A_{4}$ is self-dual), it is not hard to find the action that gives the field equations with $A_{4}$ and the fermi fields set equal to zero. It is

$$
S=\int d^{10} x \sqrt{-g}\left(R-\frac{1}{12} H_{\mu \nu \rho}^{T} \mathcal{M} H^{\mu \nu \rho}+\frac{1}{4} \operatorname{tr}\left(\partial^{\mu} \mathcal{M} \partial_{\mu} \mathcal{M}^{-1}\right)\right) .
$$

This action is manifestly invariant under global $S L(2, \mathbf{R})$ transformations. The solution we seek consists of a string-like soliton along the $x^{1}$ axis with $B$ charges $\left(q_{1}, q_{2}\right)$ and vacuum defined by $\rho(r) \sim \rho_{0}$ as $r \rightarrow \infty$, where $r^{2}=\vec{x} \cdot \vec{x}$, and $\vec{x}$ refers to the eight transverse directions $x^{2}, x^{3}, \ldots, x^{9}$. Also, the metric should approach the Minkowski metric as $r \rightarrow \infty$.

Our problem was solved some time ago by Dabholkar, et al., for a special case, namely $\vec{q}=(1,0)$ and $\rho_{0}=i$. 40 This solution, which has $\chi=0$ and $B_{\mu \nu}^{(2)}=0$, arose in considering the heterotic string, which does not contain the fields $\chi$ and $B_{\mu \nu}^{(2)}$. However, its equations 
agree with the ones being considered here when they are set to zero. Using the $S L(2, \mathbf{R})$ symmetry of the IIB theory, the solution of Dabholkar, et al., can be transformed to give a IIB solution with charges $\left(q_{1}, q_{2}\right)$ and $\rho(r) \sim \rho_{0}$. 41] The solution obtained in this way is given by

$$
\begin{gathered}
d s^{2}=A^{-3 / 4}\left(-d t^{2}+\left(d x^{1}\right)^{2}\right)+A^{1 / 4} d \vec{x} \cdot d \vec{x} \\
B_{01}^{(I)}=q_{I} \Delta_{\left(q_{1}, q_{2}\right)}^{-1 / 2} A^{-1} \\
\rho=\frac{i\left(q_{2} \chi_{0}+q_{1}\left|\rho_{0}\right|^{2}\right) A^{1 / 2}-q_{2} e^{-\phi_{0}}}{i\left(q_{1} \chi_{0}+q_{2}\right) A^{1 / 2}+q_{1} e^{-\phi_{0}}},
\end{gathered}
$$

where

$$
\begin{gathered}
A=1+\frac{Q \Delta_{\left(q_{1}, q_{2}\right)}^{1 / 2}}{r^{6}}, \\
\Delta_{\left(q_{1}, q_{2}\right)}=e^{\phi_{0}}\left|q_{1}-q_{2} \rho_{0}\right|^{2},
\end{gathered}
$$

and the charge $Q$ is a constant proportional to the tension scale $T_{1}^{(B)}$ and the $10 \mathrm{~d}$ Newton constant.

While classically $q_{1}$ and $q_{2}$ are arbitrary real numbers, quantum mechanically they must be integers. This follows (by the same reasoning Dirac used to explain the quantization of electric charge) from the existence of 5-branes and the Dirac quantization condition. Later, we will argue that stability requires that $q_{1}$ and $q_{2}$ should actually be relatively-prime integers. By allowing all pairs of relatively prime integers, we define an infinite family of string-like solitons, which form an irreducible $S L(2, \mathbf{Z})$ multiplet. Note that if, for a given string solution, $\rho_{0}$ is analytically continued outside the fundamental region $\mathcal{F}$ of $S L(2, \mathbf{Z})$, then the $S L(2, \mathbf{Z})$ transformation that brings $\rho_{0}$ back inside $\mathcal{F}$ will redefine the charges of the string.

By considering the asymptotic behavior of the metric component $g_{00}$ for $r \rightarrow \infty$, one can read off the "ADM tension" of the string 41

$$
T_{\left(q_{1}, q_{2}\right)}=\Delta_{\left(q_{1}, q_{2}\right)}^{1 / 2} T_{1}^{(B)}
$$

To get a sense of the meaning of this equation, it is convenient to restrict to the special case $\chi_{0}=0$, so that $\rho_{0}=i / \lambda_{B}$. Then the tension of the $\left(q_{1}, q_{2}\right)$ string in the canonical metric is

$$
T_{\left(q_{1}, q_{2}\right)}=\left(\lambda_{B} q_{1}^{2}+\lambda_{B}^{-1} q_{2}^{2}\right)^{1 / 2} T_{1}^{(B)} .
$$


Converting to the IIB string metric, redefines this by a factor of $\lambda_{B}^{-1 / 2}$, giving

$$
\tilde{T}_{\left(q_{1}, q_{2}\right)}=\left(q_{1}^{2}+\lambda_{B}^{-2} q_{2}^{2}\right)^{1 / 2} T_{1}^{(B)}
$$

Thus, in the string metric, the fundamental string tension is a constant, $\tilde{T}_{(1,0)}=T_{1}^{(B)}$. The $D$-string, which carries $R R$ charge only, on the other hand has tension $\tilde{T}_{(0,1)}=\lambda_{B}^{-1} T_{1}^{(B)}$. The scaling $T \sim \lambda^{-1}$ is characteristic of $D$-branes in the string metric. 42 This is to be contrasted with ordinary solitons, like the 't Hooft-Polyakov monopole, which have $T \sim \lambda^{-2}$.

As is typical of BPS mass formulas, the tensions we have found satisfy a triangle inequality

$$
T_{\left(p_{1}+q_{1}, p_{2}+q_{2}\right)} \leq T_{\left(p_{1}, p_{2}\right)}+T_{\left(q_{1}, q_{2}\right)}
$$

and equality requires that $\vec{p}$ and $\vec{q}$ are parallel. This means that if $q_{1}$ and $q_{2}$ are relatively prime, a string with charges $\left(q_{1}, q_{2}\right)$ and tension $T_{\left(q_{1}, q_{2}\right)}$ is absolutely stable, protected by charge conservation and a "tension gap" (the analog of a mass gap) from decay into multiple strings. On the other hand, a string with charges $\left(n q_{1}, n q_{2}\right)$ is at the threshold for decay into $n\left(q_{1}, q_{2}\right)$ strings. Whether one has a bound state or not, in such a case, is a delicate issue whose answer depends on the particular problem. We will show that the duality relation to M theory requires that only strings with $q_{1}$ and $q_{2}$ relatively prime be included. This conclusion is supported by a bound-state analysis carried out by Witten. [43] One way of stating the conclusion is that $q_{1}$ fundamental strings and $q_{2} D$-strings (all of which are parallel) can form a single bound state if and only if $q_{1}$ and $q_{2}$ are relatively prime. It should also be noted that the $\left(-q_{1},-q_{2}\right)$ string is the orientation-reversed $\left(q_{1}, q_{2}\right)$ string.

\subsection{Compactification of IIB Theory on a Circle}

Let us now consider type IIB string theory compactified on a circle of radius $R_{B}$ (and circumference $\left.L_{B}=2 \pi R_{B}\right)$. Since all of the $\left(q_{1}, q_{2}\right)$ strings are related by $S L(2, \mathbf{Z})$ transformations, they are all equivalent and any one of them can be weakly coupled. However, when one is weakly coupled, all the others are necessarily strongly coupled. Nevertheless, let us consider an arbitrary $\left(q_{1}, q_{2}\right)$ string and write down the spectrum of its $9 \mathrm{~d}$ excitations in the limit of weak coupling. This is given by standard string theory formulas:

$$
M_{B}^{2}=\left(\frac{m}{R_{B}}\right)^{2}+\left(2 \pi R_{B} n T_{\left(q_{1}, q_{2}\right)}\right)^{2}+4 \pi T_{\left(q_{1}, q_{2}\right)}\left(N_{L}+N_{R}\right) .
$$


Here $m$ is the Kaluza-Klein excitation number and $n$ is the string winding number. $N_{L}$ and $N_{R}$ are excitation numbers of left-moving and right-moving oscillator modes, and the level-matching condition is

$$
N_{R}-N_{L}=m n
$$

Now our purpose is to use this formula for all the $\left(q_{1}, q_{2}\right)$ strings simultaneously. However, the formula is completely meaningless at strong coupling, and (as we have said) at most one of the strings is weakly coupled. The appropriate trick in this case is to consider only BPS states - ones belonging to short supersymmetry multiplets. They are easy to identify, being given by either $N_{L}=0$ or $N_{R}=0$. (Ones with $N_{L}=N_{R}=0$ are ultrashort.) For these states the mass formula should be exact, even at strong coupling. Therefore, it can be used for all the strings at the same time. In this way, we obtain reliable mass formulas for a very large part of the spectrum - much more than appears in perturbation theory. Of course, the appearance of this rich spectrum of BPS states depends crucially on the compactification.

Using eqs. (36) and (37), the winding-mode term in eq. (41) contains the factor

$$
n^{2} \Delta_{\left(q_{1}, q_{2}\right)}=e^{\phi_{0}}\left|\ell_{1}-\ell_{2} \rho_{0}\right|^{2}
$$

where $\left(\ell_{1}, \ell_{2}\right)=n\left(q_{1}, q_{2}\right)$. There is a unique correspondence between the three integers $n, q_{1}, q_{2}$ and an arbitrary pair of integers $\ell_{1}, \ell_{2}$. The integer $n$ is the greatest common division of $\ell_{1}$ and $\ell_{2}$. The only ambiguity is whether to choose $n$ or $-n$, but since $n$ is (oriented) winding number and the $\left(-q_{1},-q_{2}\right)$ string is the orientation-reversed $\left(q_{1}, q_{2}\right)$ string, the two choices are actually equivalent. Thus BPS states are characterized by three integers $m, \ell_{1}, \ell_{2}$ and oscillator excitations corresponding to $N_{L}=|m n|$, tensored with a 16-dimensional short multiplet from the $N_{R}=0$ sector (or vice versa).

\subsection{Comparison with M Theory on a Torus}

Let us now consider 11d M theory compactified on a torus. The torus is characterized by a complex modulus $\tau=\tau_{1}+i \tau_{2}$ (as usual) and by its area $A_{M}$, measured in the $11 \mathrm{~d}$ canonical metric. If the two periods of the torus are $2 \pi R_{11}$ and $2 \pi R_{11} \tau$, then $A_{M}=\left(2 \pi R_{11}\right)^{2} \tau_{2}$. In terms of coordinates $z=x+i y$ on the torus, a single-valued wave function has the form

$$
\phi_{\ell_{1}, \ell_{2}} \sim \exp \left\{\frac{i}{R_{11}}\left[x \ell_{2}-\frac{y}{\tau_{2}}\left(\ell_{2} \tau_{1}-\ell_{1}\right)\right]\right\} .
$$


These characterize Kaluza-Klein excitations. The contribution to the mass-squared is given by the eigenvalue of $-\partial_{x}^{2}-\partial_{y}^{2}$,

$$
\frac{1}{R_{11}^{2}}\left(\ell_{2}^{2}+\frac{1}{\tau_{2}^{2}}\left(\ell_{2} \tau_{1}-\ell_{1}\right)^{2}\right)=\frac{\left|\ell_{1}-\ell_{2} \tau\right|^{2}}{\left(\tau_{2} R_{11}\right)^{2}} .
$$

Our purpose is to match BPS states of M theory on $T^{2}$ and IIB theory on $S^{1}$. Clearly, this term has the right structure to match the string winding-mode terms described at the end of the last subsection, provided that we make the identification 41, 44]

$$
\tau=\rho_{0}
$$

The normalizations of the two terms we are matching are not the same, but that is because they are measured in different metrics. The matching tells us how to relate the two metrics, a formula to be presented soon. For now, let me emphasize that the identification $\tau=\rho_{0}$ is a pleasant surprise, because it implies that the non-perturbative $S L(2, \mathbf{Z})$ symmetry of the IIB theory, after compactification on a circle, can be reinterpreted as the modular group of a toroidal compactification! Of course, once the symmetry is established for finite $R_{B}$, it should also persist in the limit $R_{B} \rightarrow \infty$.

To go further, we also need an $\mathrm{M}$ theory counterpart of the term $\left(m / R_{B}\right)^{2}$ in the IIB string mass formula. Here there is also a natural candidate: wrapping $M$ theory 2-branes so as to cover the torus $\mathrm{M}$ times. If the 2-brane tension is $T_{2}^{(M)}$, this gives a contribution $\left(A_{M} T_{2}^{(M)} m\right)^{2}$ to the mass-squared. Matching the normalization of this term, as well as the Kaluza-Klein term, one learns that the metrics are related by

$$
g^{(M)}=\beta^{2} g^{(B)}
$$

where

$$
\beta^{2}=A_{M}^{1 / 2} T_{2}^{(M)} / T_{1}^{(B)},
$$

and that the compactification volumes are related by

$$
\left(T_{1}^{(B)} L_{B}^{2}\right)^{-1}=\frac{1}{(2 \pi)^{2}} T_{2}^{(M)} A_{M}^{3 / 2}
$$

Since all the other factors are constants, this gives (for fixed $\tau=\rho_{0}$ ) the scaling law $L_{B} \sim$ $A_{M}^{-3 / 4}$. 
We still have the oscillator excitations of the type IIB string BPS mass formula to account for. Their M theory counterparts must be excitations of the wrapped 2-brane. Unfortunately, since the quantization of the 2-brane is not yet understood, this cannot be checked. The story could be turned around at this point to infer what the BPS excitation spectrum of wrapped 2-branes must be. Maybe, trying to understand this spectrum will lead to a better understanding of 2-brane quantization. In any case, assuming that this works, we have found that Kaluza-Klein excitations of the type IIB theory compactified on a circle correspond to wrappings of the 2-brane on the torus and that Kaluza-Klein modes of $\mathrm{M}$ theory on the torus correspond to windings of an infinite family of type IIB strings on the circle.

In the preceding discussion it was not specified exactly how the M theory 2-brane wraps on the torus when it "covers it $m$ times." There are a variety of different possible maps that could define the mapping, and it should be specified which ones are allowed and what is the proper way to count them. I do not have a complete answer to this question, but there is one comment that may prove useful. In the simpler problem of M theory compactified on a circle, the IIA string in 10d arises from wrapping one cycle of a toroidal 2-brane on the spatial circle. 46] If one were to wrap the circle $m$ times, instead, this would appear to give a IIA string of $m$ times the usual tension. However, it is quite clear that no such string exists, so such a configuration must be unstable to decay into $m$ strings. Perhaps the rule is that one cycle should be wrapped only once and the dual one $m$ times, but this requires identifying a preferred cycle on the torus. The only preferred cycle in the problem is the one defined by the Kaluza-Klein excitation.

\subsection{Matching p-branes}

We have conjectured that $\mathrm{M}$ theory compactified on a torus of area $A_{M}$ and modular parameter $\tau$ is identical to type IIB string theory compactified on a circle of circumference $L_{B}$ and vacuum parameter $\rho_{0}$. The conjecture was supported by matching BPS 0-branes in $9 \mathrm{~d}$, which dictated how to match parameters $\left(\tau=\rho_{0}\right.$, etc. $)$. We can carry out additional tests of the proposed duality, and learn interesting new relations at the same time, by also matching BPS $p$-branes with $p>0$ in 9d. 47. Here we will describe the results for $p=1,2,3$, 4, though other cases can also be analyzed.

Let us start with $p=1$ (strings) in $9 \mathrm{~d}$. Trivial reduction of the IIB strings in $10 \mathrm{~d}$ gives 
strings with the same charges $\left(q_{1}, q_{2}\right)$ and tensions $T_{\left(q_{1}, q_{2}\right)}$ in $9 \mathrm{~d}$. The interesting question is how these should be interpreted in $\mathrm{M}$ theory. The way to do this is to start with a 2-brane of toroidal topology in $\mathrm{M}$ theory and to wrap one of its cycles on a $\left(q_{1}, q_{2}\right)$ homology cycle of the spatial torus. The minimal length of such a cycle is

$$
L_{\left(q_{1}, q_{2}\right)}=2 \pi R_{11}\left|q_{1}-q_{2} \tau\right|=\left(A_{M} \Delta_{\left(q_{1}, q_{2}\right)}\right)^{1 / 2} .
$$

Thus, this wrapping gives a $9 \mathrm{~d}$ string whose tension is

$$
T_{\left(q_{1}, q_{2}\right)}^{(11)}=L_{\left(q_{1}, q_{2}\right)} T_{2}^{(M)}
$$

The superscript 11 emphasizes that this is measured in the $11 \mathrm{~d}$ metric. To compare with the IIB string tensions, we use eqs. (47) and (48) to deduce that

$$
T_{\left(q_{1}, q_{2}\right)}=\beta^{-2} T_{\left(q_{1}, q_{2}\right)}^{(11)}=\left(\Delta_{\left(q_{1}, q_{2}\right)}\right)^{1 / 2} T_{1}^{(B)}
$$

This agrees with the result in subsection 4, showing that this is a correct interpretation.

To match 2-branes in $9 \mathrm{~d}$ we must wrap the IIB theory 3-brane on the circle and compare to the $\mathrm{M}$ theory 2-brane. The wrapped 3-brane gives a 2-brane with tension $L_{B} T_{3}^{(B)}$. Including the metric conversion factor, the matching gives

$$
T_{2}^{(M)}=\beta^{3} L_{B} T_{3}^{(B)}
$$

Combining this with eqs. (48) and (49) gives the identity

$$
T_{3}^{(B)}=\frac{1}{2 \pi}\left(T_{1}^{(B)}\right)^{2}
$$

It is remarkable that the $\mathrm{M}$ theory/IIB theory duality not only relates $\mathrm{M}$ theory tensions to IIB theory tensions, but it implies a relation involving only IIB tensions. The 3-brane tension is a constant in the canonical metric, but using eq. (32) it scales as $\lambda_{B}^{-1}$ in the string metric, as expected for a $D$-brane.

Wrapping the $\mathrm{M}$ theory 5-brane on the spatial torus gives a $9 \mathrm{~d} 3$-brane, which can be identified with the IIB theory 3-brane reduced to 9d. This gives

$$
T_{5}^{(M)} A_{M} \beta^{4} T_{3}^{(B)}
$$


which combined with eqs. (48) and (54) implies that

$$
T_{5}^{(M)}=\frac{1}{2 \pi}\left(T_{2}^{(M)}\right)^{2} .
$$

This corresponds to satisfying the Dirac quantization condition with the minimum allowed product of charges. p

The matching of 4-branes works similarly. The IIB theory has an infinite family of 5branes with tensions $T_{5\left(q_{1}, q_{2}\right)}^{(B)}$. Wrapping a cycle on the spatial circle gives a family of $9 \mathrm{~d}$ 4-branes with tensions $L_{B} T_{5\left(q_{1}, q_{2}\right)}^{(B)}$. This should match the 4-branes obtained by wrapping the M theory 5 -brane on a $\left(q_{1}, q_{2}\right)$ homology cycle of the spatial torus. Thus,

$$
T_{5}^{(M)} L_{\left(q_{1}, q_{2}\right)}=\beta^{5} L_{B} T_{5\left(q_{1}, q_{2}\right)}^{(B)}
$$

Combined with eqs. (48), (49), (50), and (56), this implies that the IIB 5-brane tensions are given by

$$
T_{5\left(q_{1}, q_{2}\right)}^{(B)}=\frac{1}{(2 \pi)^{2}}\left(\Delta_{\left(q_{1}, q_{2}\right)}\right)^{1 / 2}\left(T_{1}^{(B)}\right)^{3} .
$$

In this case $q_{1}$ is the magnetic RR charge and $q_{2}$ is the magnetic NS-NS charge. Thus, the tension of a 5 -brane with pure $\mathrm{RR}$ charge scales as $\lambda_{B}^{1 / 2}$ and the tension of one with pure NS-NS charge scales as $\lambda_{B}^{1 / 2}$. Converting to the string metric, these become $\lambda_{B}^{-1}$ and $\lambda_{B}^{-2}$, respectively, as expected for a $D$-brane and an ordinary soliton.

The matching of 5 -branes in $9 \mathrm{~d}$ works differently. The $\mathrm{M}$ theory 5 -brane reduced to $9 \mathrm{~d}$ corresponds in the IIB picture to the Kaluza-Klein 5-brane, which couples magnetically to the $U(1)$ gauge field associated to the isometry of the circle. Similarly, the two-parameter family of IIB theory 5-branes corresponds to the two-parameter family of Kaluza-Klein 5branes, in the $\mathrm{M}$ theory description, which couple magnetically to the two $U(1)$ gauge fields associated to the two isometries of the torus.

\subsection{Implications of the Duality}

What does the IIB/M theory duality mean? Certain facts are an immediate consequence of the scaling rule $L_{B} \sim A_{M}^{-3 / 4}$. Namely, compactifying $\mathrm{M}$ theory on a torus and letting $A_{M} \rightarrow$ 0, while holding $\tau$ fixed, gives the IIB theory in 10d in the limit. Similarly, compactifying

\footnotetext{
${ }^{3}$ According to Ref. [48], it corresponds to one-half of the minimum product. The result given here has been confirmed by $D$-brane arguments, so I am quite sure it is correct.
} 
the IIB theory on a circle and letting $L_{B} \rightarrow 0$, for fixed modulus $\rho_{0}$, gives $\mathrm{M}$ theory in $11 \mathrm{~d}$ in the limit.

When $L_{B}$ and $A_{M}$ are finite, and the vacuum has only 9d Poincaré symmetry, one might ask "how many compactified dimensions are there?" From the IIB viewpoint there is one, and from the M theory viewpoint there is two. Is one of these answers better than the other? Can they be combined and regarded as three compact dimensions? To see what is happening it is instructive to list the $9 \mathrm{~d}$ massless bosonic fields showing their corresponding $\mathrm{M}$ theory and IIB descriptions.

$\begin{array}{cc}\text { M theory } & \text { IIB theory } \\ g_{\mu \nu}^{(M)} & g_{\mu \nu}^{(B)} \\ g_{\mu \alpha}^{(M)} & B_{\mu 9}^{(\alpha)} \\ g_{\alpha \beta}^{(M)} & \rho, g_{99}^{(B)} \\ C_{\mu \nu \rho} & A_{\mu \nu \rho 9} \\ C_{\mu \nu \alpha} & B_{\mu \nu}^{(\alpha)} \\ C_{\mu \nu \alpha \beta} & g_{\mu 9}^{(B)}\end{array}$

The indices $\alpha, \beta=1,2$ refer to the two internal directions of the M theory torus, the index 9 refers to the IIB theory circle, and $\mu, \nu$ are $9 \mathrm{~d}$ indices. What the list demonstrates is that which fields are "matter" and which ones are "geometrical" is subjective, depending on whether you adopt an M theory or IIB theory viewpoint. Both viewpoints are valid, and neither is preferable to the other. So, how many compact dimensions there are is just a matter of how the fields are labelled! However, there is no straightforward choice of labelling that exhibits three compact dimensions. In my opinion, some of the recent suggestions that these theories can be derived from $12 \mathrm{~d}$ are effectively counting the dimensions of both the torus and the circle.

The tests and implications of the M theory/IIB theory duality that we have presented so far are certainly not the only ones. For example, there are other solitons - intersecting p-branes, for example - that break $3 / 4$ or $7 / 8$ of the supersymmetry. 49. They still have good BPS saturation properties, so that they are under control. It would be instructive to consider the matching of these solitons in $9 \mathrm{~d}$, too, something that has not yet been done. 


\subsection{Theory/SO(32) Theory Duality}

As we mentioned at the beginning of this section, there is a second duality that is closely related (and, therefore, quite similar) to the one we have been discussing. It relates M theory compactified on $S^{1} / \mathbf{Z}_{2} \times S^{1}$ to $S O(32)$ theory compactified on $S^{1}$. [50] Since $S^{1} / \mathbf{Z}_{2}$ can be regarded as a line interval $I, S^{1} / \mathbf{Z}_{2} \times S^{1}$ can be regarded as a cylinder $C$. We will choose its height to be $L_{1}$ and its circumference to be $L_{2}=2 \pi R_{2}$. The circumference of the circle on which the $S O(32)$ theory is compactified is denoted $L_{O}=2 \pi R_{O}$ in the canonical 10d metric.

Before describing $p$-brane matching in 9d, let us briefly review the Horava-Witten picture of the $E_{8} \times E_{8}$ heterotic string theory. [26] Compactification of M theory on $S^{1} / \mathbf{Z}_{2}=I$ gives a space-time with two 10d faces, separated by a distance $L_{1} \sim \lambda_{H}^{2 / 3}$, where $\lambda_{H}$ is the coupling constant of the $E_{8} \times E_{8}$ theory in $10 \mathrm{~d}$. The two 10d faces are sometimes called "end-of-theworld 9-branes." Each of them carries the gauge fields for one of the two $E_{8}$ 's. For reasons that will be explained in the next section, $M$ theory 2-branes are allowed to terminate on a face, so that the boundary of the 2-brane is a circle inside the face. In this picture, an $E_{8} \times E_{8}$ heterotic string is a cylindrical 2-brane suspended between the two faces, with one $E_{8}$ current algebra associated to each boundary. This cylinder (or strip) is well approximated by a string living in $10 \mathrm{~d}$ when the separation $L_{1}$ is small. Since perturbation theory in $\lambda_{H}$ is an expansion about $L_{1}=0$, the fact that there really are eleven dimensions and that the string is actually a membrane is invisible in that approach.

The story described above is very similar to the relation between the IIA superstring and $\mathrm{M}$ theory. In that case the compact dimension is a circle and the IIA string arises from wrapping the $\mathrm{M}$ theory 2-brane around the circle. Thus, in a sense, the non-perturbative $E_{8} \times E_{8}$ theory just involves modding out the non-perturbative IIA theory by a $\mathbf{Z}_{2}$. (This is a bit glib, since the rules for carrying out the modding in M theory, which is not a string theory, are not so obvious.) Similarly, the type I $S O(32)$ string theory in $10 \mathrm{~d}$ can be constructed as a $\mathbf{Z}_{2}$ orientifold of the type IIB theory in 10d. Thus, the duality we are considering now can be viewed as arising from modding out the previous one by a $\mathbf{Z}_{2}$ on both sides. However, in the following we will treat it as a separate problem instead of attempting to exploit that picture. Because of the similarity of the two problems, fewer details will be provided this time. 
The $S O(32)$ theory in 10d has both a type I and a heterotic description, which are $S$ dual. That is, their coupling constants, $\lambda=e^{<\phi>}$, satisfy $\lambda_{H}^{(0)}=\left(\lambda_{I}^{(0)}\right)^{-1}$. As before, we match supersymmetry-preserving (BPS) branes in 9d. Recall that in the $S O(32)$ theory, there is just one two-form field $B_{\mu \nu}$, and the $p$-branes that couple to it are the $S O(32)$ heterotic string and its magnetic dual, which is a 5-brane. The type I open and closed strings do not carry a conserved charge and are not BPS. This is the reason that they can break. So from the type I viewpoint it is clear that the heterotic string can give a 0-brane or a 1-brane in $9 \mathrm{~d}$ and that the dual 5-brane can give a 5-brane or a 4-brane in 9d. In each case, the issue is simply whether or not one cycle wraps around the spatial circle.

Now we need to find the corresponding $9 \mathrm{~d} p$-branes from the $\mathrm{M}$ theory viewpoint, to understand why they are the only ones, and to explore what can be learned from matching tensions. We described how the $E_{8} \times E_{8}$ string arises in $10 \mathrm{~d}$ from wrapping the $\mathrm{M}$ theory 2 -brane on $I$. Subsequent reduction on a circle can clearly give a 0-brane or a 1-brane. But why is there no BPS 2-brane in 9d? When the 2-brane is forced to be in a 10d boundary, rather than in the $11 \mathrm{~d}$ bulk, it becomes breakable (non-BPS). The technical reason (see the next section) is that there is no 3-form gauge field on the boundary (or in the 10d reduction). The story for the five-brane is just the reverse. Whereas the 2-brane must wrap on the $I$ dimension, the five-brane must not do so. As a result it gives a 5-brane or a 4-brane in $9 \mathrm{~d}$ according to whether or not it wraps around the $S^{1}$ dimension. So, altogether, both pictures give the electric-magnetic dual pairs $(0,5)$ and $(1,4)$ in $9 \mathrm{~d}$.

From the $p$-brane matching one learns that

$$
\lambda_{H}^{(0)}=\frac{L_{1}}{L_{2}} .
$$

Thus, the $S O(32)$ heterotic string is weakly coupled when the spatial cylinder of the M theory compactification is a thin ribbon $\left(L_{1} \ll L_{2}\right)$. This is consistent with the earlier conclusion that the $E_{8} \times E_{8}$ heterotic string is weakly coupled when $L_{1}$ is small. Conversely, the type I string is weakly coupled for $L_{2} \ll L_{1}$, in which case the spatial cylinder is long and thin. The $\mathbf{Z}_{2}$ transformation that inverts the modulus of the cylinder, $L_{1} / L_{2}$, corresponds to the type I/heterotic duality of the $S O(32)$ theory. Since it is not a symmetry of the cylinder it implies that two different-looking string theories are $S$ dual. This is to be contrasted with the $S L(2, \mathbf{Z})$ modular group symmetry of the torus, which accounts for the self-duality of 
the IIB theory.

The $p$-brane matching in $9 \mathrm{~d}$ gives the relation

$$
L_{1} L_{2}^{2} T_{2}^{(M)}=\left(\frac{T_{1}^{(0)} L_{0}^{2}}{2 \pi}\right)^{-1},
$$

which is the analog of eq. 49. As in that case, it tells us that for fixed modulus $L_{1} / L_{2}$, one

has the scaling law $L_{O} \sim A_{C}^{-3 / 4}$, where $A_{C}=L_{1} L_{2}$ is the area of the cylinder. Eq. 56 relating $T_{2}^{(M)}$ and $T_{5}^{(M)}$ is reobtained, and one also learns that

$$
T_{5}^{(O)}=\frac{1}{(2 \pi)^{2}}\left(\frac{L_{2}}{L_{1}}\right)^{2}\left(T_{1}^{(O)}\right)^{3}
$$

In the heterotic string metric, where $T_{1}^{(O)}$ is a constant, this implies that $T_{5}^{(O)} \sim\left(\lambda_{H}^{(O)}\right)^{-2}$, as is typical of a soliton. In the type I superstring metric, on the other hand it implies that $T_{1}^{(O)} \sim 1 / \lambda_{I}^{(O)}$ and $T_{5}^{(O)} \sim 1 / \lambda_{I}^{(O)}$, consistent with the fact that both are $D$-branes from the type I viewpoint. So, what kind of object you have depends very much on your point of view.

\subsection{Some Remarks on the Origins of Chirality in M Theory}

In its heyday (around 1980) there were two major reasons for being skeptical about 11d supergravity. The first was its evident lack of renormalizability, which led to the belief that it does not approximate a well-defined quantum theory. The second was its lack of chirality (i.e., its left-right symmetry), which suggested that it could not have a vacuum with the chiral structure required for a realistic model. Our attitude towards both these issues now needs to be reconsidered. First, we now view 11d supergravity as a low-energy effective description of $\mathrm{M}$ theory. As such, it seems reasonable to believe that there is a well-defined quantum interpretation. The situation with regard to chirality is also changed. Here the new ingredients are the branes - the 2-brane and 5-brane, as well as the end-of-theworld 9-branes. They can and do introduce left-right asymmetry (consistent with anomaly cancellation requirements).

In the duality between $\mathrm{M}$ theory on a torus and IIB theory on a circle, that we have been discussing, the issue of chirality already appears. In the limit that the area $A_{M}$ of the torus vanishes, one obtains the IIB theory in $10 \mathrm{~d}$, which is a chiral theory. Let's track down the 
M theory origins of the chiral asymmetry. One question is whether it is property of the limit $A_{M} \rightarrow 0$, or whether it is already visible in $9 \mathrm{~d}$. Let's look at this question first from the IIB viewpoint.

The chiral fields of the IIB theory in $10 \mathrm{~d}$ are the massless fermions and the four-form $A_{4}$, whose field strength is self-dual. This means that the associated physical degrees of freedom belong to parity non-invariant representations of the massless little group, spin (8). Compactifying on a circle, they give BPS Kaluza-Klein towers of excitations with $M_{n}^{2}=$ $\left(n / R_{B}\right)^{2}$. These belong now to representations of the massive $9 \mathrm{~d}$ little group, which is also spin (8). Indeed, it is obvious that these are the same parity non-invariant representations we started with. So, in this sense, one could say that these massive excitations are chiral. Certainly, they account for the chirality of the massless 10d field in the decompactification limit. The massive 4-form modes in $9 \mathrm{~d}$ are described by complex fields (combining $n$ and $-n$ ). Dropping the index $n$, they satisfy a free wave equation of the form $A_{4} \sim i m * F_{5}$. 51] Taking the exterior derivative, $F_{5} \sim i m d\left(* F_{5}\right)$. Even though there is no manifestly covariant action in $10 \mathrm{~d}$ for a 4 -form with a self-dual field strength, there is one for this massive complex 4-form in $9 \mathrm{~d}$ :

$$
\mathcal{L}\left(A_{4}\right) \sim \int F_{5}^{*} \cdot F_{5} d^{9} x+i m \int A_{4}^{*} \wedge F_{5}
$$

As expected, this has a parity-violating mass term. The number of propagating modes described by such a Lagrangian is the same for $m \neq 0$ as it is for $m=0$. This structure is quite similar to the much-studied "topologically massive gauge theory" in 3d: 52

$$
\mathcal{L}\left(A_{1}\right) \sim \int F_{2} \cdot F_{2} d^{3} x+m \int A_{1} \wedge F_{2}
$$

One difference is that the construction can be carried out for real fields in dimensions $4 k-1$ whereas complex fields are required in dimensions $4 k+1$.

Having identified where the chirality resides in 9d, we can now ask how these states originate in the $11 \mathrm{~d}$ description. The answer is immediate, because one of the things we learned from studying the M theory/IIB theory duality is that type IIB Kaluza-Klein excitations on $S^{1}$ correspond to $\mathrm{M}$ theory wrapping modes of the 2-brane on $T^{2}$. Therefore, the massive chiral modes in $9 \mathrm{~d}$ must arise from wrapping the 2-brane. The 2-brane world-volume theory itself is $3 \mathrm{~d}$, so one might think it could not be chiral. However, it contains a "charge" coupling to the background 3-form gauge field $\int C_{\mu \nu \rho} d x^{\mu} \wedge d x^{\nu} \wedge d x^{\rho}$, and this is precisely the 
relevant chiral term. The 3 -form gives a $U(1)$ gauge field in $9 \mathrm{~d}$ with two indices in internal directions $\left(C_{\mu 12}\right)$, and the massive chiral modes due to $n$ units of wrapping carry $n$ units of electric charge, as measured by this gauge field in $9 \mathrm{~d}$. The corresponding field in the IIB picture is the Kaluza-Klein gauge field $g_{\mu 9}$. (This correspondence already appeared in eq. (59).)

\section{WHICH BRANES CAN END ON WHICH?}

In considering the Horava-Witten description of the $E_{8} \times E_{8}$ heterotic string, we concluded that in $\mathrm{M}$ theory a 2-brane can terminate on an end-of-the-world 9-brane. This section discusses, with several examples, the general question of when one supersymmetric (BPS) brane is allowed to terminate on another one. Following Strominger, we argue that charge conservation is an essential consideration. 53. However, we will discover that there is also a subtle "wormhole" construction, which gives additional possibilities. The basic idea is explained by $D$-branes, which are defined as $p$-branes on which strings can terminate. 42. The type II (A or B) fundamental string carries a conserved charge and couples electrically to the NS-NS sector two-form gauge field $B_{\mu \nu}$. When a string carrying this kind of conserved charge has an end, flux associated with a $U(1)$ gauge field emerges from the end into the $D$ brane. This means that there is a point electric charge on the end of the string (explaining Chan-Paton factors), and that the $D$-brane world-volume theory contains a $U(1)$ gauge field $A_{\alpha}$, which can assume a suitable configuration to carry away the flux. An interesting generalization is the case of $N$ coincident $D$-branes when the $[U(1)]^{N}$ gauge symmetry of the individual $D$-branes gets extended to a non-Abelian $U(N)$ gauge symmetry. Let us turn now to specific examples of branes ending on branes.

\subsection{Three Examples}

An example of a $D$-brane is the 2-brane of type IIA string theory in 10d. As with all type II $D$-branes, massless fields of the world-volume theory form an $N=110 \mathrm{~d}$ gauge multiplet, restricted to the brane. The vector $A_{\mu}$ of the $10 \mathrm{~d}$ gauge theory decomposes into a three-vector $A_{\alpha}$ and seven scalars $\phi_{i}$ in the world-volume theory. The scalars can be regarded as collective coordinates for excitations of the brane in the seven transverse dimensions or as Goldstone 
bosons for broken translational symmetries. Similarly, the world-sheet fermions corrspond to broken supersymmetries. The gauge field $A_{\alpha}$ carries off the flux when a type IIA string terminates on the 2-brane. From the point of view of this gauge field, the charge on the end of the string is electric. However, there is a dual magnetic picture, which is also interesting. The world-volume theory of the 2-brane can be recast by a duality transformation $\left(d A=* d \phi_{8}\right)$ that replaces $A_{\alpha}$ by an eighth scalar $\phi_{8}$.54, 55] This scalar is a zero-form gauge field, and from its point of view the charge on the end of the string is magnetic. However, a more profound viewpoint is that $\phi_{8}$ represents excitations in an eighth transverse dimension, so that the 2-brane actually lives in an 11d space-time. This strongly suggests that, after the duality transformation, one is describing the 2-brane of $M$ theory. But this raises a paradox: M theory in 11d Minkowski space does not have strings that can terminate on the 2-brane, so what is the strong coupling description of a configuration consisting of a type IIA string ending a 2-brane? I'll return to this question later in this section.

Let's now consider the Dirichlet 5-brane of the type IIB theory. By the same reasoning as before, the $6 \mathrm{~d}$ world-volume theory in this case contains a six-vector $A_{\alpha}$ and four scalars $\phi_{i}$ representing transverse excitations. Let us once again replace the $U(1)$ gauge field $A_{\alpha}$ by a dual gauge field. In $6 \mathrm{~d}$ the dual gauge field is a three-form. As explained in Section 2.2, a 2-brane can couple electrically to a three-form. Thus, BPS 2-branes can live inside the 5-brane. What this means is that the 3-brane of the IIB theory can terminate on the 5-brane. 53 Its boundary is a 2-brane, and the charge that exists on its boundary gives rise to electric flux of the three-form gauge field of the 5-brane. Equivalently, had we not made a duality transformation, it would give magnetic flux of the original $U(1)$ gauge field. Thus, it is consistent with charge conservation for a type IIB 3-brane to terminate on a Dirichlet 5-brane. The $S L(2, \mathbf{Z})$ duality symmetry of the type IIB theory can be invoked to draw additional conclusions. Under an $S L(2, \mathbf{Z})$ transformation the 3-brane is invariant, but the Dirichlet 5-brane, which carries $B_{\mu \nu}^{(I)}$ magnetic charges $(0,1)$, can be transformed into a $\left(q_{1}, q_{2}\right) 5$-brane. This implies that the 3 -brane is allowed to terminate on any of the 5-branes.

As a third example, let us consider the $\mathrm{M}$ theory 5-brane. Its massless sector consists of a $6 \mathrm{~d} N=2$ tensor supermultiplet. [56, 57 The bosons in this multiplet are a two-form gauge field $B_{\alpha \beta}$, with a self-dual field strength $(d B=-* d B)$, and five scalars $\phi_{i}$ describing transverse excitations of the 5 -brane in $11 \mathrm{~d}$. The fact that this is the appropriate multiplet 
can be argued in many different ways. Here we will simply remark that this is the only matter supermultiplet with the correct supersymmetry, and it contains the desired number of scalar fields. The two-form can couple to a self-dual string, which can be identified as the boundary of 2-brane that ends on the 5-brane. Thus, the $\mathrm{M}$ theory 2-brane can terminate on the M theory 5-brane, but not on another 2-brane. Thus, the M theory 5-brane can be regarded as a higher-dimension analog of a $D$-brane. Rather than being defined as an object on which an open string can end, it is an object on which an open membrane can terminate. The reason that the $D$-brane picture is so powerful is that open strings can be quantized, and they can be used to describe excitations of the $D$-brane. We do not have this kind of mathematical control for open membranes, so the $D$-brane picture is less useful (at the present time) in the case of $\mathrm{M}$ theory. However, compactification on a circle makes it clear that this is really more than an analogy. If the compactification is arranged so that one dimension of both the 2-brane and the 5-brane are wrapped on the spatial circle, then the resulting $10 \mathrm{~d}$ picture precisely corresponds to a IIA string ending on a IIA 4-brane. This 4-brane is a standard $D$-brane.

\subsection{Parallel p-branes}

When a $p_{1}$-brane is allowed to end on a $p_{2}$-brane, then it is also possible to consider a pair of parallel $p_{2}$ branes with an open $p_{1}$-brane suspended between them. To be clear what we are talking about, let me emphasize that all the branes under consideration are supersymmetric (BPS) branes carrying conserved charges. This means (for $p_{2}<D-3$ ) that a pair of parallel $p_{2}$-branes (infinite hyperplanes) is a stable configuration because the forces between the branes cancel in such a case. If one imagines attaching an open $p_{1}$-brane that connects them, its tension would cause some bending of the $p_{2}$-branes in the vicinity of the junction. As far as I know, explicit field configurations that realize this picture have not been studied, but the qualitative picture is clear. In any case, the main reason to be interested in such configurations is as a way of thinking about quantum excitations of a system of parallel p-branes. New classical configurations are more of a curiosity.

As the separation of two $p_{2}$-branes becomes small, the "length" $\ell$ of the $p_{1}$-brane becomes

small. In this case, it can become a good approximation to view the pair of $p_{2}$-branes (and the intervening space) as a single $p_{2}$-dimensional system and the collapsed $p_{1}$-brane as a 
$\left(p_{1}-1\right)$-brane of tension $T_{N C}=\ell T_{p_{1}}$ inside this $p_{2}$-dimensional space. The subscript $\mathrm{NC}$ denotes 'non-critical', since the tension of such branes can be arbitrarily small (as $\ell \rightarrow 0$ ). Note that in the case of the $E_{8} \times E_{8}$ heterotic string the vanishing of the tension as $\ell \rightarrow 0$ can be compensated by a Weyl rescaling of the metric so that there is a finite tension in the limit. In other cases, such as the ones to be discussed here, such a rescaling is not appropriate, and the non-critical $\left(p_{1}-1\right)$-brane has a spectrum of excitations that become massless as $\ell \rightarrow 0$. These excitations should be viewed as possible excitations of a pair of nearly coincident $p_{2}$-branes.

A word of warning is in order here. The solitons that are being described as " $p$ dimensional" are given by field configurations that spread to some extent in the transverse dimensions. As explained to me by Maldacena, in certain cases the limit in which the length $\ell \rightarrow 0$ can result in the size of transverse spread becoming large at the same time. Then the simple geometric picture becomes misleading. Most of the considerations that follow do not require taking a limit $\ell \rightarrow 0$, so they are not subject to this criticism. The limit only appears when one wants to identify non-critical branes.

The specific example that we will focus on here is a pair of parallel 3-branes in $9 \mathrm{~d}$. Our purpose in doing this is to extract additional implications of the duality between $\mathrm{M}$ theory compactified on a torus and type IIB theory compactified on a circle. We have already seen that a single 3-brane in $9 \mathrm{~d}$ can be viewed equally as a IIB 3-brane or as an M-theory 5-brane wrapped on the spatial torus. Now we wish to extend this picture to a pair of parallel 3branes including the possibility of suspending other branes between them. The plan is to first consider parallel 3-branes of the IIB theory and then parallel 5-branes of M theory.

\subsection{Parallel 3-branes of Type IIB Theory}

Since 3 -branes of IIB theory are $D$-branes, fundamental $(1,0)$ type IIB strings can end on them. Also, such a string can be suspended between a pair of parallel 3-branes. The 4d worldvolume theory of this system is a $U(2)$ gauge theory, spontaneously broken to $U(1) \times U(1)$ when the separation $\ell>0$. The open string introduces a unit of electric charge for a $U(1)$ subgroup. (The appropriate $U(1)$ is the one inside the $S U(2)$ factor.) The sign of the charge is tied to the orientation of the string. An $S L(2, \mathbf{Z})$ duality transformation gives the same

configuration with the fundamental $(1,0)$ string replaced by a $\left(q_{1}, q_{2}\right)$ string. In this case the 
lightest modes have mass

$$
M=\ell T_{\left(q_{1}, q_{2}\right)}=\ell\left(q_{1}^{2}+\lambda_{B}^{-2} q_{2}^{2}\right)^{1 / 2} T_{1}^{(B)}
$$

where we have used the results of Section 2.4 for the tension of $\left(q_{1}, q_{2}\right)$ strings. This formula, which can be generalized to include a $\theta$ angle, agrees with the BPS formula for dyons of the $N=4$ gauge theory in $4 \mathrm{~d}$. Thus, this picture relates the conjectured $S L(2, \mathbf{Z})$ duality of $N=4$ gauge theory in $4 \mathrm{~d}$ to that of type IIB superstring theory in 10d. Note that in this example it is 0 -branes that are becoming massless as $\ell \rightarrow 0$. This implies that only states with spin $J \leq 1$ have $M \rightarrow 0$ as $\ell \rightarrow 0$. This picture is also applicable in $9 \mathrm{~d}$ if a dimension orthogonal to the brane is compactified.

\subsection{Parallel 5-branes of M Theory}

As we have explained, an M theory 2-brane can end on a 5-brane, and, therefore, a 2-brane can be suspended between a pair of parallel 5-branes. To make contact with the results in preceding subsection, we wish to take this configuration and compactify on a spatial torus in such a way that we end up with a string suspended between 3-branes in 9d. The interesting point is that this can be done such that the string is a $\left(q_{1}, q_{2}\right)$ string. This is to be expected because the torus is responsible for the $S L(2, \mathbf{Z})$ duality.

To give a specific realization suppose that the 5-brane spatial coordinates are in the $x_{2}, x_{3}, x_{4}, x_{5}, x_{6}$ hyperplane, and the other coordinates are fixed at two sets of values on the two 5-branes. Now suppose that the $x_{2}$ and $x_{3}$ coordinates are compactified to form the spatial torus. This implies that the 5-branes are wrapped on the torus, giving parallel 3branes in $9 \mathrm{~d}$. Now consider a 2-brane connecting the two 5-branes. To get a string in $9 \mathrm{~d}$ we want only one of its coordinates to be wrapped. Therefore, suppose it lies in a plane defined by $x_{1}, x_{2}^{\prime}$, with the other coordinates fixed. Here $x_{2}^{\prime}$ is a line in the $x_{2}-x_{3}$ plane, and hence on the spatial torus. It belongs to the $\left(q_{1}, q_{2}\right)$ homology class if it satisfies $q_{2} x_{2}=q_{1} x_{3}$. In this way, we obtain what we wanted, a $\left(q_{1}, q_{2}\right)$ string suspended between parallel 3-branes in $9 \mathrm{~d}$. This is the dual description of the configuration obtained in the preceding subsection 


\subsection{A Paradox and its Resolution}

In the preceding subsection we considered an M theory 2-brane suspended between parallel 5 -branes and showed that it could be wrapped on a spatial torus to give a $\left(q_{1}, q_{2}\right)$ string suspended between parallel 3-branes. An alternative possibility would be to wrap two dimensions of the 5-branes and no dimensions of the 2-brane on the spatial torus. This gives a 2-brane suspended between parallel 3-branes in 9d. What is the IIB interpretation of this configuration? The only simple guess is an open 3-brane suspended between parallel 3-branes. This is not allowed, however, because the boundary of a 3-brane is a 2-brane and the world-volume theory does not contain the gauge field needed to carry off its flux. Before explaining the right answer, let us first examine an analogous problem for which the answer is known.

The IIA theory in 10d can have a string suspended between a pair of parallel 4-branes, since they are $D$-branes. As we remarked earlier, this is interpreted in $\mathrm{M}$ theory as a wrapping of an 11d configuration consisting of a 2-brane suspended between parallel 5-branes. But what is the $\mathrm{M}$ theory interpretation of a string suspended between parallel 2-branes? The two problems seem quite analogous, but the interpretations are rather different. The simplest guess, an open 2-brane suspended between parallel 2-branes is not allowed by charge conservation, so that is not the answer. The correct answer, which we will now describe, was presented by Aharony, Sonnenschein, and Yankielowicz. [58. (I first heard it from Polchinski. Later, Yankielowicz explained it to me in detail and drew my attention to ref. [58.)

Consider an M theory 2-brane in a "wormhole" configuration. This means one smooth surface consisting of parallel 2-branes connected by a throat . As described, there are actually two anti-parallel branes connected by a throat. Such a configuration is highly unstable. The size of the throat would grow rapidly eating up the branes. To stabilize the configuration one must flip-over one of the two faces to make them parallel. This can be achieved by rotating one of them by $\pi$ in a plane orthogonal to the throat - the $x_{3}-x_{4}$ plane, say. This rotation involves no self-intersections of the surface, but since more than three spatial dimensions are required, it is somewhat difficult to visualize. In any case, after doing this we have two parallel branes connected by a throat. This configuration is consistent with charge conservation and represents an excitation of a BPS system. 
Now the desired 10d configuration - an open string suspended between parallel 2-branes - can be obtained by compactification on a circle. The geometry is a bit subtle, however. Suppose $y$ is the compact coordinate of the spatial circle, and $x_{2}$ and $x_{3}$ are the coordinates that parametrize one of the faces. Now imagine going around a circle on the face that encloses the throat. By continuity with the connecting tube (the throat), which is wrapped around the $y$ direction, it is clear that this circle also winds around the $y$ direction. The coordinate $y$ corresponds to the scalar field of the M theory 2-brane world volume, which was called $\phi_{8}$ earlier when we introduced it as a zero-form dual of a $U(1)$ gauge field. The throat is a source of $y$ (or $\phi_{8}$ ) magnetic charge on the end faces, since $\oint d y \neq 0$ when the contour encloses the throat. In the dual formulation, appropriate to the IIA description of the membrane, $\phi_{8}$ is replaced by a $U(1)$ gauge field $A_{\alpha}$ and this magnetic charge is re-interpreted as electric charge. This matches the picture expected from the $D$-brane viewpoint, showing that we have found the correct $\mathrm{M}$ theory interpretation.

Now it is clear what the answer to the original problem - the IIB interpretation of a 2-brane suspended between parallel 3-branes in $9 \mathrm{~d}$ - should be. The correct 10d picture is a parallel 3-branes connected by a throat, altogether forming one smooth surface. Again one of the 3-branes must be flipped over to ensure that they are parallel and not anti-parallel. Compactification on a circle is then arranged so that one dimension of the connecting throat is wrapped leaving a 2-brane from the $9 \mathrm{~d}$ viewpoint. The throat again introduces a magnetic charge $\oint d y$. In $9 \mathrm{~d}$ this is best viewed in the dual picture in which $y$ is replaced by a two-form, since the 3-brane has a $4 \mathrm{~d}$ world volume. A two-form is just what is needed to carry off the flux associated to the charge on the string-like boundary of a 2-brane. So, again, there is a consistent picture.

It was emphasized earlier that the $N=4$ gauge theory that describes the world-volume theory of a pair of parallel 3-branes has an infinite spectrum of dyons with $J \leq 1$ whose mass vanishes as $\ell \rightarrow 0$. They were interpreted in terms of strings suspended between the 3-branes. Now we have seen that in 9d there can also be a suspended 2-brane, which gives a noncritical string as $\ell \rightarrow 0$. The string modes describe states of arbitrarily high spin whose mass vanishes as $\ell \rightarrow 0$ in the effective $N=44 \mathrm{~d}$ gauge theory. 


\subsection{Three-String Junctions}

The definition of a $D$-brane as a $p$-brane on which a type II string can end has to be interpreted carefully for $p \leq 1$. For example, in the case of IIB strings, we found that there is an infinite family of strings with $B_{\mu \nu}$ charges $\left(q_{1}, q_{2}\right)$, where $q_{1}$ and $q_{2}$ are relatively prime integers. The $(1,0)$ string is the fundamental string and the $(0,1)$ string is the $D$-string. A naive interpretation of 'a fundamental string ending on a $D$-string' is described as the junction of three string segments, one of which is $(1,0)$ and two of which are $(0,1)$. This is not correct, however, because the charge on the end of the fundamental string results in flux that must go into one or the other of the attached string segments changing the string charge in the process. In short, the three-string junction must satisfy charge conservation. 58 This means that an allowed junction that describes the joining of three strings has charges $\left(q_{1}^{(i)}, q_{2}^{(i)}\right), i=1,2,3$ such that $q_{1}^{(i)}$ and $q_{2}^{(i)}$ are relatively prime for each value of $i$ and $\sum_{i} q_{1}^{(i)}=\sum_{i} q_{2}^{(i)}=0$. The configuration is stable if the three strings are semi-infinite and the angles are chosen so that tensions, treated as vectors, add to zero. It would be interesting to construct the corresponding solution of the supergravity field equations. I expect it to be supersymmetric and, therefore, to have the usual nice BPS properties. For example, a periodic array could be formed so that compactification on a circle would give the same configuration in $9 \mathrm{~d}$.

Given the three-string junction in $9 \mathrm{~d}$, it is natural to ask about its $11 \mathrm{~d} \mathrm{M}$ theory interpretation. The answer is easily found and very pleasing. In $11 \mathrm{~d}$ one could consider a three-membrane junction which consists of a single smooth surface. Topologically it is the same as the "pants diagram," which describes the world sheet for a closed string breaking into two closed strings. Of course, in the present problem the time coordinate is suppressed and the surface is just a spatial diagram (like real pants).

Now we want to compactify on a torus to $9 \mathrm{~d}$ in such a way that one dimension of each of the three protruding 2-branes is wrapped on the torus. Labelling the three 2-branes by an index $i=1,2,3$, we want the circular coordinate of the $i$ th 2-brane to wrap on a $\left(q_{1}^{(i)}, q_{2}^{(i)}\right)$ homology cycle of the torus. It is a simple geometrical fact that this is only possible if $\sum_{i} q_{1}^{(i)}=\sum_{i} q_{2}^{(i)}=0$. Thus, we reproduce the three-string junction in $9 \mathrm{~d}$ with exactly the desired properties. 
This three-string junction could prove to be useful in future studies. For example, one might wish to consider a collection of wrapped, intersecting 3-branes as part of a study of black holes in string theory. Excitations of such a system would be represented by open strings connecting the 3-branes. However, it would also be possible to have open strings ending on three different 3-branes if they are joined by a junction of the kind we have described. Of course, given the existence of the three-string junction, one can also build up more complicated networks, similar to $\phi^{3}$ Feynman diagrams.

\section{6D STRING VACUA WITH EXTENDED SUPER- SYMMETRY}

Compactification to $9 \mathrm{~d}$ was sufficient to demonstrate that there are dualities connecting all superstring theories to one another as well as to $\mathrm{M}$ theory. Also, we learned that the dualities in $9 \mathrm{~d}$ provide non-perturbative information about these theories. In this section we will survey many (possibly all) superstring vacua with extended supersymmetry in 6d. The next section will give a much less complete survey of $6 \mathrm{~d}$ vacua with $\mathrm{N}=1$ supersymmetry. In the process, many new dualities that give additional insights into the structure of the theory will appear.

Since vacua are characterized by their unbroken supersymmetry and massless spectrum, among other things, we need to know what the possibilities are in 6d. Massless particles are specified by representations of the little group, which is spin (4) or $S U(2) \times S U(2)$. Labelling representations by $S U(2) \times S U(2)$ multiplicities, one can have the following massless particles

$$
\begin{aligned}
\text { graviton : } & (3,3) \\
\text { gravitino : } & 2(3,2) \text { or } 2(2,3) \\
\text { self dual tensor : } & (3,1) \text { or }(1,3) \\
\text { vector : } & (2,2) \\
\text { spinor : } & 2(2,1) \text { or } 2(1,2) \\
\text { scalar : } & (1,1) .
\end{aligned}
$$

Parity interchanges the two $S U(2)$ 's. Thus, the fermions and self-dual tensors are chiral, while the other particles are not. The factors of two in the fermion multiplicities appear because a Weyl spinor in $6 \mathrm{~d}$ is necessarily complex. The supersymmetry type can be labelled by the number of gravitinos of each chirality. The maximal case (32 supercharges) corresponds 
to $(2,2)$ or $N=4$ and is non-chiral. There are two $N=2$ possibilities, just as in ten dimensions: $(1,1)$ or IIA is non-chiral and $(2,0)$ or IIB is chiral. $N=1$ or $(1,0)$ supersymmetry is also chiral. One might also consider $(4,0),(3,1),(3,0)$, and $(2,1)$ supersymmetries, but I believe that none of these is possible.f

\subsection{Vacua with $(2,2)$ Supersymmetry}

Let us begin with maximal $(2,2)$ unbroken supersymmetry. In this case there is a unique massless multiplet - the supergravity multiplet. One way to determine its particle is by decomposing the massless fields of $11 \mathrm{~d}$ supergravity into $6 \mathrm{~d}$ pieces. The result is as follows:

$$
\begin{aligned}
\text { bosons: } & (3,3)+5(3,1)+5(1,3)+16(2,2)+25(1,1) \\
\text { fermions : } & 4(3,2)+4(2,3)+10(2,1)+10(1,2) .
\end{aligned}
$$

The $U$ duality group in this case is $S O(5,5 ; \mathbf{Z})$ and the moduli space, parametrized by the 25 scalar fields, is $\mathcal{M}_{5,5}$.

A string vacuum with this amount of supersymmetry and this massless sector can be obtained either by compactifying the type IIB theory on $T^{4}$ or $\mathrm{M}$ theory on $T^{5}$. Each construction explains a subgroup of the $U$ duality group. The type IIB theory compactification gives a $T$ duality group $S O(4,4 ; \mathbf{Z})$ from the $T^{4}$; in the M theory compactification, the modular group of $T^{5}$ accounts for an $S L(5, \mathbf{Z})$. The complete answer, $S O(5,5 ; \mathbf{Z})$, is the smallest group that contains both of these as subgroups. Once again, we see the power of M theory/IIB theory duality. By considering both at the same time one can make deductions that are not apparent from either viewpoint separately. The multiplicities of the other boson fields are given by $S O(5,5)$ representations. Thus, for example, the 16 vectors belong to a spinor representation, which is real for this signature. The 10 tensors $((3,1)$ and $(1,3))$ belong to the fundamental representation of $S O(5,5)$. The fact that three-form field strengths mix with their duals under the duality group is a higher-dimension analog of electric-magnetic duality, and is a hallmark of an $S$ duality. The fermions belong to representations of the denominator algebra $-S O(5) \times S O(5)$ in this case - in constructions of this type.

\footnotetext{
${ }^{4}$ A gravity supermultiplet has been proposed for the $(2,1)$ case, [59 but it gives gravitational anomalies that cannot be cancelled.
} 


\subsection{Vacua with $(1,1)$ Supersymmetry}

Let us now consider vacua with non-chiral $2 \mathrm{~A}$ supersymmetry. In this case the gravity supermultiplet consists of

$$
\begin{aligned}
\text { bosons : } & (3,3)+(3,1)+(1,3)+4(2,2)+(1,1) \\
\text { fermions : } & 2(3,2)+2(2,3)+2(2,1)+2(1,2) .
\end{aligned}
$$

In addition there can be massless vector supermultiplets, whose content is

$$
(2,2)+4(1,1)+2(2,1)+2(1,2)
$$

When there are $n$ Abelian vector multiplets, so that the gauge group is $[U(1)]^{n}$, the duality group is $S O(4, n ; \mathbf{Z})$, and the moduli space $\mathcal{M}_{4, n}$ is spanned by the $4 n$ scalar fields belonging to the vector multiplets. The additional scalar field in the supergravity multiplet, which can be identified as the dilaton, has a moduli space $\mathbf{R}$, so that the complete moduli space is $\mathcal{M}_{4, n} \times \mathbf{R}$.

Toroidal compactification of the heterotic string to $6 \mathrm{~d}$ gives a vacuum with $(1,1)$ supersymmetry and $n=20$, which is the standard Narain result. [7] Note that the compactification of 20 left-moving dimensions gives rise to the 20 vector fields belonging to the vector supermultiplets, whereas the compactification of 4 right-moving dimensions gives the 4 vector fields belonging to the supergravity multiplet.

The heterotic string vacuum described above (with $n=20$ ) has a dual description given by the type IIA string compactified on $K 3$. [5] We won't present a complete discussion of this result, which will be discussed by other lecturers, but simply verify that the massless field content works out correctly. Compactification on $K 3$ breaks half the supersymmetry that is present in 10d, giving the desired $(1,1)$ supersymmetry, so it suffices to check the bosonic field content. To do this, we need some basic facts about the cohomology and the moduli space of $K 3$. As usual for a compact connected 4 -manifold, $b_{0}=1$ and $b_{4}=1$. Furthermore $b_{1}=b_{3}=0$, so the only non-trivial cohomology is $H^{2}$. It has 22 generators, which can be chosen to be 3 self-dual 2 -forms $\left(b_{2}^{+}=3\right)$ and 19 anti-self-dual 2 -forms $\left(b_{2}^{-}=19\right)$. The moduli space of complex structure deformations, for a $K 3$ of fixed volume, is $\mathcal{M}_{3,19}$, which is 57 dimensional. Including the volume of the manifold, there are 58 moduli altogether. This implies that the $6 \mathrm{~d}$ zero modes obtained form the $10 \mathrm{~d}$ metric consist of the $6 \mathrm{~d}$ metric and 
58 scalar fields. Altogether, the massless bosonic IIA fields on $K 3$ give

$$
\begin{aligned}
g_{\mu \nu} & \rightarrow(3,3)+58(1,1) \\
\phi & \rightarrow(1,1) \\
A_{\mu} & \rightarrow(2,2) \\
B_{\mu \nu} & \rightarrow(3,1)+(1,3)+22(1,1) \\
C_{\mu \nu \rho} & \rightarrow 23(2,2) .
\end{aligned}
$$

One of the 23 vectors obtained from $C_{\mu \nu \rho}$ arises because a 3-form in $6 \mathrm{~d}$ is equivalent (by a duality transformation) to a vector. These multiplicities agree precisely with those of $(1,1)$ supergravity coupled to 20 vector supermultiplets.

The $6 \mathrm{~d}$ duality between the heterotic string on $T^{4}$ and the IIA string on $K 3$ can be lifted to a $7 \mathrm{~d}$ duality. 23] Since the IIA theory can be viewed as M theory on $S^{1}$, it is plausible that one can identify this $S^{1}$ with one of the $S^{1}$ 's inside the $T^{4}$ used to compactify the heterotic theory. Decompactifying this $S^{1}$ then leaves a $7 \mathrm{~d}$ duality between $\mathrm{M}$ theory compactified on $K 3$ and the heterotic theory compactified on $T^{3}$. This duality also passes all the checks that have been made. For example, the duality group computed from both viewpoints is $S O(3,19 ; \mathbf{Z})$ and the moduli space is $\mathcal{M}_{3,19} \times \mathbf{R}$.

It is even possible to go one more step and to lift this duality to 8d using "F theory." 39. This topic is beyond the scope of these lectures, so let's just state the result. On the one hand, consider the heterotic string compactified on $T^{2}$, which has a duality group $S O(2,18 ; \mathbf{Z})$ and a moduli space $\mathcal{M}_{2,18} \times \mathbf{R}$. The $\mathrm{F}$ theory dual description consists of a non-perturbative type IIB vacuum, which can be formally described as a compactification from $12 \mathrm{~d}$ to $8 \mathrm{~d}$ on a special class of $K 3$ manifolds - those that have an elliptic fibration. Remarkably, the moduli space of these $K 3$ 's is given by a $\mathcal{M}_{2,18}$ subspace of the $\mathcal{M}_{3,19}$ moduli space of $K 3$ 's, so that the matching of moduli spaces required for the duality again works beautifully.

The $6 \mathrm{~d}$ duality between the heterotic string theory on $T^{4}$ and the IIA string theory on $K 3$ is an $S$ duality. One way to see this is by comparing low-energy effective supergravity Lagrangians and noting that the field mapping between the two descriptions includes

$$
\phi_{H}=-\phi_{I I A}
$$

as well as a duality transformation of the two-form potential. This relation between dilaton fields implies that the coupling constants are reciprocal to one another, which is $S$ duality. This also means that the heterotic and type IIA strings, both of which occur in the $6 \mathrm{~d}$ theory, 
are electric-magnetic duals of one another. When one is regarded as fundamental, the other must be viewed as a soliton. This observation is the key to understanding their 10d origins. In the 10d IIA theory the magnetic dual of the IIA string is the 5-brane. Thus, the heterotic string in $6 \mathrm{~d}$ arises from wrapping a 5 -brane of topology $K 3 \times S^{1}$ on the spatial $K 3$, leaving a string $\left(S^{1}\right)$ in 6d. 60, 61, 62 The corresponding BPS soliton of the 6d supergravity field equations has been constructed explicitly. 63, 61] The converse story, which must surely be true too, is less well established. It requires that the IIA string in $6 \mathrm{~d}$ should arise from wrapping a 5-brane of the 10d heterotic theory on the spatial $T^{4}$.

We have now found two entirely different constructions of heterotic strings as $M$ theory solitons. The first one (discussed in Section 2.9) arises from wrapping the M theory 2-brane on $S^{1} / \mathbf{Z}_{2}=I$. The second one, which we have just found, entails wrapping the $\mathrm{M}$ theory 5 -brane on $K 3$. Later, we will discuss a class of vacua in which both kinds of heterotic strings can occur at the same time.

In the heterotic picture, perturbative reasoning shows that there is enhanced gauge symmetry at singular points of the moduli space $\mathcal{M}_{4,20}$. It is interesting to ask where the additional massless states come from in the dual description. The mechanism, which is nonperturbative, is that the singular points of the moduli space correspond to limits in which a two-cycle on the $K 3$ shrinks to a point. [23, 64] Type IIA 2-branes wrapped on the two-cycle give 0 -branes in $6 \mathrm{~d}$ whose mass is proportional to the area of the two cycle. There is an ADE classification of the two-cycles on $K 3$ that can vanish, which has just the properties required to account for the symmetry enhancement that is obtained in the dual heterotic description.

We have explained that a type IIA vacuum in $6 \mathrm{~d}$ should have a duality group $S O(4, n ; \mathbf{Z})$ and a moduli space $\mathcal{M}_{4, n} \times \mathbf{R}$, and then we presented a pair of dual constructions for the special case $n=20$. It is natural to ask whether other values of $n$ are also possible. Constructions that give other values of $n$ are conveniently described from the M theory viewpoint. The $n=20$ result, itself, can be viewed as arising from $\mathrm{M}$ theory compactified on $K 3 \times S^{1}$, since type IIA theory is M theory compactified on $S^{1}$. To generalize this, the idea is to replace the compact space by $\left(K 3 \times S^{1}\right) / \mathbf{Z}_{h}$. For this to work, one needs to restrict to a class of $K 3$ 's having a $\mathbf{Z}_{h}$ discrete symmetry, and then to combine the action of the generator of this group with a rotation by $2 \pi / h$ on the circle. This ensures that there are no fixed points, so that a smooth manifold results. The $h=2$ case was analyzed in ref. 65. The 
possible discrete symmetries of $K 3$ 's have been classified by the mathematician Nikulin. 66. Chaudhuri and Lowe have applied his results to the problem at hand to conclude that the complete set of possibilities is given by 67]

$$
\begin{aligned}
h=2 & \rightarrow n=12 \\
h=3 & \rightarrow n=8 \\
h=4 & \rightarrow n=6 \\
h=5,6 & \rightarrow n=4 \\
h=7,8 & \rightarrow n=2 .
\end{aligned}
$$

Thus, there are consistent string vacua for these values of $n$. The $h=5$ and 6 constructions give the same massless spectrum and moduli space, but it is not known whether they are completely identical. (The same remark applies to $h=7,8$.) It is also not known whether other $n$ values could be obtained by other constructions that have not yet been considered.

In the special case of $h=2$ a dual construction is known. 68 One starts with the $E_{8} \times E_{8}$ heterotic string compactified on $T^{4}$, and mods out by a $\mathbf{Z}_{2}$ that interchanges the two $E_{8}$ 's. Clearly, this reduces the rank by 8 (reducing $n$ from 20 to 12).

\subsection{Vacua with $(2,0)$ Supersymmetry}

Type IIB supersymmetry in 6d admits two massless supermultiplets. The gravity supermultiplet particle content is

$$
(3,3)+4(2,3)+5(1,3)
$$

and the tensor supermultiplet particle content is

$$
(3,1)+4(2,1)+5(1,1)
$$

A superstring vacuum with IIB supersymmetry in 6d can be obtained by compactifying the type IIB superstring on $K 3$. The resulting massless bosons in $6 \mathrm{~d}$ are as follows:

$$
\begin{aligned}
g_{\mu \nu} & \rightarrow(3,3)+58(1,1) \\
\phi, \chi & \rightarrow 2(1,1) \\
B_{\mu \nu}^{(1)}, B_{\mu \nu}^{(2)} & \rightarrow 2(3,1)+2(1,3)+44(1,1) \\
A_{\mu \nu \rho \lambda}^{+} & \rightarrow 3(1,3)+19(3,1)+(1,1) .
\end{aligned}
$$

This shows that the massless content is given by the gravity multiplet plus 21 tensor multiplets. 
Since the particle content is chiral, there are potential gravitational anomalies. In six dimensions these are characterized by eight-forms. Up to a common overall normalization, the contributions of each of the chiral fields is as follows:

$$
\begin{gathered}
2(2,3): \frac{49}{72} \operatorname{tr} R^{4}-\frac{43}{288}\left(\operatorname{tr} R^{2}\right)^{2} \\
2(1,2): \frac{1}{360} \operatorname{tr} R^{4}+\frac{1}{288}\left(\operatorname{tr} R^{2}\right)^{2} \\
(1,3): \frac{7}{90} \operatorname{tr} R^{4}-\frac{1}{36}\left(\operatorname{tr} R^{2}\right)^{2}
\end{gathered}
$$

Combining these with weights corresponding to one gravity multiplet and $n_{T}$ tensor multiplets, one finds that the sum vanishes provided that $n_{T}=21$. Thus, $n_{T}=21$, the result we found by $K 3$ compactification of the IIB superstring, is the only value that can give a consistent anomaly-free theory. 69

The vacua with $n_{T}=21$ have the duality group $S O(5,21 ; \mathbf{Z})$ and the moduli space $\mathcal{M}_{5,21}$ parametrized by the 105 scalars in the tensor multiplets. Note that there are no scalars in the gravity supermultiplet, so that this is the complete moduli space. This also means that the dilaton must be one of these 105 scalar fields. Compactifying further on a circle to $5 \mathrm{~d}$ gives one more scalar field corresponding to the radius of the circle. There are no other new scalars, since the $6 \mathrm{~d}$ theory has no vector fields. Then the moduli space becomes $\mathcal{M}_{5,21} \times \mathbf{R}$. This $5 \mathrm{~d}$ model has the same supersymmetry, massless fields, and moduli space as the heterotic string compactified to $5 \mathrm{~d}$, so it is natural to conjecture that they are dual. In fact, this is a consequence of two dualities that we have already discussed: Type IIA on $K 3 \sim$ heterotic on $T^{4}$ and IIA on $S^{1} \sim$ IIB on $S^{1}$. In the heterotic picture, the scalar field that corresponds to the $\mathbf{R}$ factor in the moduli space is the dilaton. Thus, we have a $U$ duality: the heterotic string compactified on $T^{5}$ at strong coupling corresponds to the type IIB string on $K 3 \times S^{1}$ at large radius of the $S^{1}$. In particular, the strong-coupling limit of the $5 \mathrm{~d}$ heterotic string is six dimensional. This is analogous, of course, to the fact that the strong-coupling limit of the $10 \mathrm{~d}$ type IIA string or $E_{8} \times E_{8}$ heterotic string is eleven dimensional.

The $6 \mathrm{~d}$ theory with IIB supersymmetry and 21 tensor supermultiplets has another dual description given by $M$ theory compactified on the orbifold $T^{5} / \mathbf{Z}_{2}$. [70, 71] The $\mathbf{Z}_{2}$ acts on each of the five circles of the torus, which introduces 32 orbifold points. Including the other six space-time dimensions, they are 32 orbifold planes. In units where an M theory 
5-brane carries one unit of magnetic charge, it turns out that each of these orbifold planes carries $-1 / 2$ unit of magnetic charge. The charge cannot be cancelled locally, but it can be cancelled globally by introducing 165 -branes. This is necessary, since the total charge on a compact space must vanish. One can then account for the massless field content as follows: Compactification of $11 \mathrm{~d}$ supergravity on $T^{5} / \mathbf{Z}_{2}$ gives an untwisted sector consisting of the gravity multiplet and five tensor multiplets. Each of the 5-branes introduces an additional tensor multiplet, so that altogether there are 21 of them, as required. The 5-branes can be represented as points on the $T^{5} / \mathbf{Z}_{2}$, and their coordinates are controlled by the five scalar fields in the tensor multiplet associated to the 5-brane. The 25 scalar fields belonging to the other five tensor multiplets arise from zero modes of the $11 \mathrm{~d}$ metric and three-form on $T^{5}$.

The $6 \mathrm{~d}$ theory contains, among other things, self-dual solitonic strings whose tension can become arbitrarily small in suitable limits. In terms of the IIB superstring compactified on $K 3$, their appearance can be traced to singular limits in which a two-cycle on the $K 3$ shrinks to a point. 72. The reason for this is that the self-dual 3-brane can wrap around the cycle, leaving a string in the $6 \mathrm{~d}$ space-time whose tension is proportional to the area of the two-cycle. In the IIA case, the corresponding mechanism gave 0-branes with an ADE classification. Here it gives non-critical strings with an ADE classification. The existence of strings whose tension can become small, so that they effectively decouple from gravity, is an interesting phenomenon. It would be very desirable to have a better understanding of their properties, because they seem to encode in a deep way the essential features of $N=4$ gauge theory. The point is that, compactifying further to $4 \mathrm{~d}$ on $T^{2}$, windings of the string around the two cycles of the torus give electric and magnetic charge in $4 \mathrm{~d}$. 73 The $S L(2, \mathbf{Z})$ duality of $N=4$ gauge theory derives from that of the torus (again!). The appearance of non-critical strings can also be understood in terms of the description in terms of $\mathrm{M}$ theory compactified on $T^{5} / \mathbf{Z}_{2} \cdot 71$ In this case, there are 16 "parallel" 5-branes, represented by points on the compact space. Since an M theory 2-brane can be suspended between parallel 5 -branes, when a pair of 5-branes approach one another this 2-brane is approximated by a string whose tension is proportional to the separation of the 5-branes. 


\section{6DSTRING VACUA WITH N = 1 SUPERSYMME- TRY}

The preceding section described various possibilities for superstring vacua with extended supersymmetry in $6 \mathrm{~d}$. The supersymmetry was very constraining, so the classification presented was reasonably complete. In the case of $N=1$ supersymmetry in $6 \mathrm{~d}$ the story becomes much more complex. Each time the number of supersymmetries or the number of uncompactified dimensions is decreased, new issues arise. While our ultimate goal is to understand vacua with $N=1$ or $N=0$ in $4 \mathrm{~d}$, I am most comfortable proceeding in steps, absorbing the lessons at one stage before moving on to the next one. The cutting edge, where the understanding is increasing most rapidly at the present time, is for vacua with $N=1$ in 6 d or with $N=2$ supersymmetry in 4 d. I will only discuss the former, and even this will not be complete. Many, but not all, $4 \mathrm{~d}$ vacua with $N=2$ can be obtained from these by a subsequent $T^{2}$ compactification.

$N=1$ supersymmetry in $6 \mathrm{~d}$ admits four kinds of massless supermultiplets:

$$
\begin{aligned}
\text { gravity : } & (3,3)+2(2,3)+(1,3) \\
\text { tensor : } & (3,1)+2(2,1)+(1,1) \\
\text { vector : } & (2,2)+2(1,2) \\
\text { hyper : } & 2(2,1)+4(1,1) .
\end{aligned}
$$

In general, a $6 \mathrm{~d} N=1$ string vacuum will give one gravity multiplet, $n_{T}$ tensor multiplets, $n_{V}$ vector multiplets, and $n_{H}$ hyper multiplets. Since all models of this kind are chiral, anomaly cancellation always provides non-trivial constraints. 34, 74 For example, cancellation of the $\operatorname{tr} R^{4}$ term in the anomaly eight-form gives the requirement

$$
n_{H}+29 n_{T}=n_{V}+273
$$

In this section we will discuss $N=1$ models constructed in a number of different ways. One approach is compactification of the $S O(32)$ theory on a smooth $K 3$. Such models can have non-perturbative symmetry enhancement when 5-branes are included. A second approach is $K 3$ compactification of the $E_{8} \times E_{8}$ theory. Non-perturbatively, this can be regarded as M theory compactified on $K 3 \times S^{1} / \mathbf{Z}_{2}$. In this case there is freedom associated with dividing the instanton number between the two $E_{8}$ 's as well as the possibility of including 5-branes. A third approach that we will mention, which turns out to be dual to one of 
the $E_{8} \times E_{8}$ compactifications, is based on $T^{4} / \mathbf{Z}_{2}$ orbifold compactification of the $S O(32)$ theory.

\subsection{General Considerations}

For the most part we will consider models with $n_{T}=1$, in which case eq. (78) simplifies to $n_{H}=n_{V}+244$. When $n_{T}=1$ it is possible to give a manifestly covariant effective action for the massless modes. The point is that the two-form with self-dual field strength in the gravity multiplet and the two form with anti-self-dual field strength in the tensor multiplet can be combined and represented by a two-form with an unconstrained field strength. Another advantage of $n_{T}=1$ is that anomaly cancellation can be achieved by straightforward analogs of the techniques introduced for 10d models in Ref. [34]. (Otherwise, a generalization given in Ref. [75] is required.) In the $n_{T}=1$ case, with a semi-simple gauge group $G=\prod G_{\alpha}$, anomaly cancellation is possible if the anomaly eight-form factorizes into a product of two four-forms. This means that $I_{8} \sim X_{4} \wedge \tilde{X}_{4}$, where

$$
\begin{aligned}
& X_{4}=\operatorname{tr} R^{2}-\sum_{\alpha} v_{\alpha} \operatorname{tr} F_{\alpha}^{2} \\
& \tilde{X}_{4}=\operatorname{tr} R^{2}-\sum_{\alpha} \tilde{v}_{\alpha} \operatorname{tr} F_{\alpha}^{2} .
\end{aligned}
$$

Here $F_{\alpha}$ is the Yang-Mills two-form associated to the group $G_{\alpha}$, given by matrices in a convenient (fundamental, for instance) representation of the Lie algebra. The $v_{\alpha}, \tilde{v}_{\alpha}$ are numerical constants.

Anomaly cancellation is achieved by assigning non-trivial Yang-Mills and local Lorentz gauge transformation assignments to the two-form $B_{\mu \nu}$, choosing its field strength to be gauge invariant ( $H=d B+$ Chern-Simons terms), and adding a suitable counterterm of the form $\int B \wedge \tilde{X}_{4}$ to the effective action. Taking the exterior derivative of the field strength $H$ gives the Bianchi identity $d H \sim \operatorname{tr} R^{2}-\sum v_{\alpha} \operatorname{tr} F_{\alpha}^{2}$. Under the $S$-duality transformation

$$
\phi \rightarrow-\phi, \quad H \rightarrow e^{-2 \phi} * H
$$

the Bianchi identity is intercharged with the one-loop corrected field equations

$$
d\left(e^{-2 \phi} * H\right) \sim \operatorname{tr} R^{2}-\sum \tilde{v}_{\alpha} \operatorname{tr} F_{\alpha}^{2}
$$


When there are also $U(1)$ factors in the gauge group, there can be additional terms in the anomaly eight-form of the structure $F \wedge Y_{6}$, where $F$ is the $U(1)$ field-strength twoform and $Y_{6}$ is a six-form. When such terms appear, anomaly cancellation can still be achieved provided there is a suitable scalar field $\chi$ that transforms under the $U(1)$ gauge transformation $(\chi \rightarrow \chi+\Lambda)$. In this case its gauge-invariant field strength has the structure $d \chi-A$. This results in the $U(1)$ gauge field eating the scalar $\chi$ to become massive. Then there is no longer an unbroken $U(1)$ gauge symmetry, but at least the theory is consistent. There is an analogous mechanism in $4 \mathrm{~d}$, which has been known for a long time. 76] However, in $4 \mathrm{~d}$ a scalar is dual to a 2 -form, so that this is just a dual description of the same mechanism as in $10 \mathrm{~d}$. In $6 \mathrm{~d}$ that is not the case.

The constants $v_{\alpha}$ in the form-form $X_{4}$ have a simple interpretation, pointed out in Ref. [77], provided the group $\prod G_{\alpha}$ can be realized by a perturbative heterotic string construction. In this case the factor $G_{\alpha}$ is realized in the world-sheet theory as an affine Kac-Moody Lie algebra. For a level $n_{\alpha}$ representation $v_{\alpha}$ is given by

$$
v_{\alpha} \operatorname{tr} F_{\alpha}^{2}=\frac{n_{\alpha}}{h_{\alpha}} \operatorname{Tr} F_{\alpha}^{2}
$$

We use the symbol 'tr' for traces in the fundamental representation and ' $T r$ ' for traces in the adjoint representation. Here, $h_{\alpha}$ is the dual Coxeter number of the group $G_{\alpha}$. In practice, the only cases we will encounter are at level one $\left(n_{\alpha}=1\right)$. In this case one can show that $v=2$ for an $S U(n)$ or $S p(n)$ group, $v=1$ for an $S O(n)$ group, $v=1 / 3$ for $E_{6}, v=1 / 6$ for $E_{7}$, and $v=1 / 30$ for $E_{8}$.

We will mostly be interested here in $K 3$ compactifications. In this case, integrating the four-form Bianchi identity over the $K 3$ manifold gives a consistency condition for the compactification. Specifically, in the $S O(32)$ case, one obtains the condition

$$
n_{1}+n_{5}=24
$$

Here, 24 arises is the Euler number of the $K 3$ manifold and $n_{1}$ is the number of instantons embedded in the $S O(32)$ gauge group, (i.e., the second Chern class of the gauge bundle). The integer $n_{5}$ is the number of 5 -branes in the solution. These 5-branes correspond to delta-function sources in $d H$ at isolated points on the $K 3$, filling the $6 \mathrm{~d}$ space-time. Their appearance is a non-perturbative phenomenon. In the case of $E_{8} \times E_{8}$ models compactified 
on $K 3$, the integrated Bianchi identity gives a very similar consistency condition

$$
n_{1}+n_{2}+n_{5}=24
$$

Here $n_{1}$ and $n_{2}$ denote the number of instantons embedded in each of the two $E_{8}$ factors, and $n_{5}$ is again the number of (non-perturbative) 5-branes. The study of branes in Section 2 showed that 5-branes of M theory (or $E_{8} \times E_{8}$ theory) and 5-branes of $S O(32)$ theory are quite different. This will be reflected here by the fact that inclusion of 5-branes has very different implications for the $6 \mathrm{~d}$ vacua in the two cases.

\subsection{K3 Compactification of the SO(32) Theory}

The $S O(32)$ theory can be viewed either as a heterotic string theory or a type I string theory since the two descriptions are $S$ dual. To make contact with the interpretation of the constants $v_{\alpha}$ in the preceding subsection, the heterotic interpretation is appropriate. Let us begin with perturbative vacua with $n_{1}=24$ and $n_{5}=0$, which were understood a long time ago. 74

To describe the instantons in the $S O(32)$ gauge group, one must select an $S U(2)$ subgroup in which to embed them. One choice (but not the only possible one, as we will see later) is to consider the decomposition $S O(32) \supset S O(28) \times S U(2) \times S U(2)$ and embed the instantons in one of the two $S U(2)$ 's. This leaves an unbroken $S O(28) \times S U(2)$ gauge symmetry. Using appropriate index theorems, one can compute the number of hypermultiplet zero modes belonging to each representation of this group. Such an analysis gives the hypermultiplet content $10(\mathbf{2 8}, \mathbf{2})+65(\mathbf{1}, \mathbf{1})$. Note that altogether there are $\frac{1}{2} 28 \cdot 27+3=381$ vector multiplets and $560+65=625$ hyper multiplets, which satisfies the condition $n_{H}=n_{V}+244$. The contribution of each of these fields to the anomaly polynomial can be computed using formulas in Refs. [74, 78, 79]. One finds

$$
\begin{gathered}
X_{4}=\operatorname{tr} R^{2}-\operatorname{tr} F_{1}^{2}-2 \operatorname{tr} F_{2}^{2} \\
\tilde{X}_{4}=\operatorname{tr} R^{2}+2 \operatorname{tr} F_{1}^{2}-44 \operatorname{tr} F_{2}^{2} .
\end{gathered}
$$

Note that $v_{1}=1$ for the $S O(28)$ factor and $v_{2}=2$ for the $S U(2)$ factor, as expected for levelone representations. The potential of scalar fields has many flat directions. At special values 
one can get symmetry enhancement, the maximal case being $S O(28) \times[S U(2)]^{6}$. However, the generic situation is for symmetry breaking ("Higgsing") to occur. The gauge symmetry can be broken to $S O(8)$ but not further.

Let us now go beyond perturbation theory and include 5-branes in the vacuum configuration. This problem was studied first by Witten, who identified the 5-branes as instantons that have shrunk to zero size, what he called "small instantons." 80 From the type I viewpoint the $S O(32)$ 5-branes (as well as the heterotic strings) are $D$-branes. In a type II theory a single $D$-brane carries a $U(1)$ gauge symmetry, and when $n$ of them coincide the group is enhanced to $U(n)$. However, the projections that give a type I theory modify these rules. For example, the 32 coincident 9-branes that fill the space-time are responsible for the $S O(32)$ gauge symmetry from the type I viewpoint. Dynamical 5-branes in the type I theory correspond to a group of four stuck together from the type II viewpoint. (This is the minimal unit, as long as the compactification manifold is smooth.) It turns out that such a dynamical 5-brane carries a $S p(1)=S U(2)$ gauge group, and that when $n$ of them coincide the symmetry is enhanced to $S p(n)$. [80] The number $n_{5}$ in the condition $n_{1}+n_{5}=24$ refers to the number of dynamical 5-branes, and so the maximum number allowed is 24 .

The example with the largest gauge group is achieved by taking $n_{1}=0$ and $n_{5}=24$ and then taking the 245 -branes to coincide. This means that they are all at the same point on the $K 3$ manifold. In this case the unbroken gauge symmetry in $6 \mathrm{~d}$ is $G=S O(32) \times S p(24)$. This group has rank 40, which is the world record for 6d models, as far as I know. The massless spectrum of this theory contains vector multiplets belonging to $S O(32) \times S p(24)$. From the type I viewpoint, the $S O(32)$ vector multiplets arise as zero modes of 99 open strings, i.e., open strings connecting 9-branes to 9-branes. Similarly, the $S p(24)$ vector multiplets arise from 55 open strings, strings connecting 5 -branes to 5 -branes. The massless hyper multiplets turn out to be as follows: 55 strings give an $S p(24)$ antisymmetric tensor $(\mathbf{1}, \mathbf{1 1 2 7})+(\mathbf{1}, \mathbf{1})$; 59 strings give $\frac{1}{2}(\mathbf{3 2}, \mathbf{4 8})$; the $K 3$ moduli give $20(\mathbf{1}, \mathbf{1})$. The factor of $1 / 2$ appears because it is possible to have "half hyper multiplets" when they belong to a pseudoreal representation of a symmetry group. (Fundamental representations of symplectic groups are pseudoreal.) Altogether there are 1672 vector multiplets and 1916 hypermultiplets, which again satisfies the condition $n_{H}=n_{V}+244$. 
The factorized anomaly polynomial of the $S O(32) \times S p(24)$ model has $[79$

$$
\begin{gathered}
X_{4}=\operatorname{tr} R^{2}-\operatorname{tr} F_{1}^{2} \\
\tilde{X}_{4}=\operatorname{tr} R^{2}+2 \operatorname{tr} F_{1}^{2}-2 \operatorname{tr} F_{2}^{2} .
\end{gathered}
$$

The point to be noted is that the $S O(32)$ group has a perturbative heterotic string interpretation, and it appears in $X_{4}$ with $v_{1}=1$, as expected. The $S p(24)$ group, on the other hand, is non-perturbative from the heterotic string viewpoint, and it does not appear in $X_{4}$.

The "small instanton" model described above can be generalized in two ways. One is to consider $n_{5}<24$ and to embed $n_{1}=24-n_{5}$ units of instanton number in the $S O(32)$ group. In this case the maximal non-perturbative gauge group is $S p\left(n_{5}\right)$. The second generalization is to allow the $n_{5} 5$-branes to come apart into groups of $\left\{n_{5 i}\right\}$ with $\sum n_{5 i}=n_{5}$. Then the non-perturbative gauge group is $\prod S p\left(n_{5 i}\right)$.

We have discussed the significance of the $v_{\alpha}$ 's in $X_{4}$, but not the $\tilde{v}_{\alpha}$ 's in $\tilde{X}_{4}$. Sagnotti has shown that supersymmetry considerations imply that the kinetic terms of the gauge fields have the form 75

$$
\sum_{\alpha}\left(v_{\alpha} e^{-\phi}+\tilde{v}_{\alpha} e^{\phi}\right) \operatorname{tr}\left(F_{\alpha} \cdot F_{\alpha}\right),
$$

where $\phi$ is the heterotic dilaton. At weak coupling $(\phi \rightarrow-\infty)$ the perturbative $v_{\alpha}$ term dominates. However, as the coupling is increased the second term becomes important. In particular, if it happens that $\tilde{v}_{\alpha}<0$, then there is a singularity (divergent coupling constant) at $\phi=\phi_{0}$, where

$$
e^{2 \phi_{0}}=-v_{\alpha} / \tilde{v}_{\alpha}
$$

Duff and collaborators have argued that this singularity is associated with the vanishing of a string tension. [81] Specifically, they argue that there are solitonic dyonic strings in 6d whose electric and magnetic charges $(p, q)$ are proportional to $\left(v_{\alpha}, \tilde{v}_{\alpha}\right)$ and whose tension is given by $T_{(p, q)}=p e^{-\phi}+q e^{\phi}$. Thus, the tension of such a string vanishes at the singularity. In the examples that have been discussed so far the $S O(n)$ group has $v_{1}=1$ and $\tilde{v}_{1}=-2$, and, therefore, its coupling constant diverges for $e^{2 \phi_{0}}=1 / 2=-p / q$. Thus, a $(1,-2)$ string becomes tensionless. These theories are well-defined for weak coupling $\left(e^{\phi_{0}} \ll 1\right)$ and should continue smoothly up to the singularity at $e^{2 \phi_{0}}=1 / 2$. At that point one expects a phase 
transition to take place. Beyond that point, our formulas are no longer applicable. Later, we will speculate about what happens at the phase transition.

\subsection{K3 Compactification of the $\mathrm{E}_{8} \times \mathrm{E}_{8}$ Theory}

Perturbative vacua of the $E_{8} \times E_{8}$ heterotic string compactified on $K 3$ have $n_{5}=0$ (no 5 -branes) and $n_{1}+n_{2}=24$. Let us begin by considering the special case $n_{1}=24, n_{2}=0$, which corresponds to embedding all 24 units of instanton number into one of the two $E_{8}$ factors. A maximal subgroup of $E_{8}$ is $E_{7} \times S U(2)$. So if we embed the instantons in this $S U(2)$, this leaves an unbroken $E_{8} \times E_{7}$ gauge symmetry. In this case application of index theorems give massless hypermultiplets transforming as $10(\mathbf{1}, \mathbf{5 6})+65(\mathbf{1}, \mathbf{1})$. Just as in the $S O(28) \times S U(2)$ model of the preceding subsection, there are 381 vector multiplets and 625 hypermultiplets. The factorized anomaly eight-form has

$$
\begin{gathered}
X_{4}=\operatorname{tr} R^{2}-\frac{1}{30} \operatorname{tr} F_{1}^{2}-\frac{1}{6} \operatorname{tr} F_{2}^{2} \\
\tilde{X}_{4}=\operatorname{tr} R^{2}+\frac{1}{5} \operatorname{tr} F_{1}^{2}-\operatorname{tr} F_{2}^{2} .
\end{gathered}
$$

Note that $v_{1}=1 / 30$ and $v_{2}=1 / 6$ are the values expected for $E_{8}$ and $E_{7}$, respectively. By giving vevs to scalars corresponding to flat directions one can find enhanced gauge symmetry as large as $E_{8} \times E_{7} \times[S U(2)]^{5}$, or symmetry breaking giving a complete Higgsing of the $E_{7}$ leaving only an unbroken $E_{8}$. Note that the $E_{8}$ kinetic term becomes singular for $\phi \rightarrow \phi_{0}$, where $e^{2 \phi_{0}}=1 / 6$. At this point a $(1,-6)$ string becomes tensionless.

The second possibility for perturbative vacua of the $E_{8} \times E_{8}$ heterotic string theory on $K 3$ is to embed some instantons in each of the $E_{8}$ 's. It is not possible to have $n=1,2,3$, so the possibilities are

$$
n_{1} \geq n_{2}=24-n_{1} \geq 4
$$

Embedding all instantons in $S U(2)$ subgroups can leave an unbroken $E_{7} \times E_{7}$. The hy-

permultiplets in this case are $\frac{1}{2}\left(n_{1}-4\right)(\mathbf{5 6}, \mathbf{1})+\frac{1}{2}\left(n_{2}-4\right)(\mathbf{1}, \mathbf{5 6})+62(\mathbf{1}, \mathbf{1})$. Half integer coefficients are allowed, because the $\mathbf{5 6}$ is a pseudoreal representation of $E_{7}$. The factorized anomaly polynomial is now

$$
X_{4}=\operatorname{tr} R^{2}-\frac{1}{6} \operatorname{tr} F_{1}^{2}-\frac{1}{6} \operatorname{tr} F_{2}^{2}
$$




$$
\tilde{X}_{4}=\operatorname{tr} R^{2}+\left(1-\frac{n_{1}}{12}\right) \operatorname{tr} F_{1}^{2}+\left(1-\frac{n_{2}}{12}\right) \operatorname{tr} F_{2}^{2} .
$$

It is interesting to ask whether any of these models could be a dual description of the perturbative $S O(32)$ string compactification. Certainly the groups we have found in the two cases are different. However, in the case of the $S O(32)$ model it was possible to Higgs to an $S O(8)$ subgroup leaving $X_{4}=\operatorname{tr} R^{2}-\operatorname{tr} F^{2}$ and $\tilde{X}_{4}=\operatorname{tr} R^{2}+2 \operatorname{tr} F^{2}$, where $F$ refers to the $S O(8)$. In the case of the $E_{7} \times E_{7}$ models under consideration here, it is possible to completely Higgs one $E_{7}$ and to Higgs the other to $S O(8)$. Then, using the rule $\operatorname{tr}_{E_{7}} F^{2} \rightarrow 6 \operatorname{tr}_{S O(8)} F^{2}$, we are left with $X_{4}=\operatorname{tr} R^{2}-\operatorname{tr} F^{2}$ and $\tilde{X}_{4}=\operatorname{tr} R^{2}+\left(6-\frac{1}{2} n_{2}\right) \operatorname{tr} F^{2}$. Thus, the two $S O(8)$ models have the same anomaly polynomials (and the same massless field content) for $n_{1}=16$, $n_{2}=8$. Thus, it is plausible (and supported by other studies) that perturbative $S O(32)$ compactifications and perturbative $(16,8) E_{8} \times E_{8}$ compactifications on $K 3$ give the same moduli space of models. Of course, the portion of the moduli space that is visible in each approach is different. To find $S O(28)$ from the $E_{8} \times E_{8}$ approach or $E_{7} \times E_{7}$ from the $S O(32)$ approach would require discovering the appropriate unHiggsing.

Now, let us move beyond perturbation theory and consider $E_{8} \times E_{8}$ models with 5branes. The 5 -branes of $E_{8} \times E_{8}$ are the 5 -branes of $\mathrm{M}$ theory, which we saw carry a $(2,0)$ tensor multiplet. But the compactification on $K 3$ cuts the supersymmetry in half leaving $N=1$. The $(2,0)$ tensor multiplet decomposes into a $N=1$ tensor multiplet plus a $N=1$ hypermultiplet. This is to be contrasted with the $S O(32) 5$-branes, which carry a $(1,1)$ vector multiplet (that decomposes into a $N=1$ vector multiplet and a $N=1$ hypermultiplet). So $S O(32)$ 5-branes carry vector multiplets, and that is why we found that they give rise to additional (non-perturbative) gauge symmetry. The $E_{8} \times E_{8} 5$-branes, on the other hand, do not have vector multiplets and they do not give additional gauge symmetry. Rather, each $E_{8} \times E_{8} 5$-brane adds a tensor multiplet (and a hypermultiplet). Thus, by including them, we obtain models with $n_{T}=n_{5}+1$ tensor multiplets. When $S O(32) 5$-branes coincided, we found that the 55 strings connecting them gave massless gauge bosons resulting in enhanced gauge symmetry. When $E_{8} \times E_{8} 5$-branes coincide, the 2-branes connecting them give tensionless strings. 82, 83

Let's begin with the extreme case $n_{5}=24, n_{1}=n_{2}=0$. 83. Since there are no instantons to embed, the $E_{8} \times E_{8}$ gauge symmetry is unbroken (so that $n_{V}=496$ ). The number of 
tensor multiplets is $n_{T}=25$. The 245 -branes each give a hypermultiplet, and there are also 20 of them associated to the $K 3$ moduli, so $n_{H}=44$. Note that these numbers satisfy the anomaly condition $n_{H}+29 n_{T}=n_{V}+273$.

The $E_{8} \times E_{8}$ theory - viewed as M theory with two boundaries - has no anomalies in the bulk (where it is non-chiral), only on the boundaries. 26] The anomaly cancellation condition, therefore, requires that the anomaly form be expressible as a sum of two factorized pieces, one associated to each boundary. This structure, which persists after $K 3$ compactification, was analyzed by Seiberg and Witten. 83 They found, in general, that for $n_{1}+n_{2} \leq 24$ and $n_{5}=24-n_{1}-n_{2}$ the anomaly polynomial can be written in the form

$$
\begin{aligned}
& \left(\frac{1}{2} \operatorname{tr} R^{2}-A_{1}\right)\left(\frac{n_{1}-8}{4} \operatorname{tr} R^{2}-\frac{n_{1}-12}{2} A_{1}\right) \\
& +\left(\frac{1}{2} \operatorname{tr} R^{2}-A_{2}\right)\left(\frac{n_{2}-8}{4} \operatorname{tr} R^{2}-\frac{n_{2}-12}{2} A_{2}\right),
\end{aligned}
$$

where

$$
A_{i}=\sum_{\alpha} v_{\alpha i} \operatorname{tr} F_{\alpha i}^{2}
$$

Here $\left(n_{1}, A_{1}\right)$ and $\left(n_{2}, A_{2}\right)$ are associated to the two boundaries. Remarkably, when $n_{1}+n_{2}=$ 24 , so that $n_{T}=1$, this can be recast as a single factorized expression.

One would like to have a global view of the moduli space of $N=1$ vacua in $6 \mathrm{~d}$. On the face of it, it would seem that there should (at least) be a separate component for each possible number of tensor multiplets $n_{T} \leq 25$. The reason is that a tensor multiplet contains a tensor field $B_{\mu \nu}^{-}$with an anti-self-dual field strength, and the only way such a field can acquire mass is by joining up with another tensor field $B_{\mu \nu}^{+}$, whose field strength is self-dual. However, the only massless $B_{\mu \nu}^{+}$belongs to the gravity supermultiplet, and it must stay put if the supersymmetry doesn't change. This simple argument can be evaded, but this requires something remarkable to happen.

In the $\mathrm{M}$ theory picture of the $E_{8} \times E_{8}$ theory, we have argued that extra tensor multiplets correspond to 5 -branes in the bulk. One could imagine the number of 5-branes changing by emission or absorption by an end-of-the-world 9-brane. 83 Inside the 9-brane it can presumably turn into an instanton. To see the transition, one should consider a 5-brane in the bulk very close to one of the 9-branes. In this case a 2-brane which can be suspended between them, is approximated by a string whose tension vanishes as the 5 -brane approaches 
the 9-brane. So once again the proposed phase transition is associated with the appearance of a string of vanishing tension. 84] This is just what one needs to evade the argument in the preceding paragraph. When a string goes to zero tension, all its modes go to zero mass, and this undoubtedly includes an infinite number of massive $B_{\mu \nu}$ fields. This makes it possible for the $B_{\mu \nu}^{-}$of the tensor multiplet to pair up with a $B_{\mu \nu}^{+}$. The idea is that $B_{\mu \nu}^{(n)}$ breaks up into $B_{\mu \nu}^{(n)+}$ and $B_{\mu \nu}^{(n)-}$, but then, on the other side of the transition, $B_{\mu \nu}^{(n)-}$ joins up with $B_{\mu \nu}^{(n+1)+}$ to become massive again, leaving $B_{\mu \nu}^{(1)+}$ available to pair with $B_{\mu \nu}^{-}$from the tensor multiplet. In view of the physical picture of the transition in terms of 5-brane emmission and absorption from 9-branes it seems likely that this actually happens and so there might be a single connected moduli space of $N=1$ vacua in 6 d. Altogether, as required by eq. (78), the massless tensor multiplet is replaced by 29 massless hypermultiplets.

\subsection{Models Without Phase Transitions}

We have seen that there are singularities, associated with the appearance of tensionless strings, at specific value of the dilaton whenever one of the $\tilde{v}_{\alpha}$ parameters is negative. Indeed this phenomenon occurs in almost all the models we have considered. However, referring to eq. (96), there are no negative $\tilde{v}_{\alpha}$ 's for the special case $n_{1}=n_{2}=12$. Thus the $(12,12)$ models, in which the instantons are embedded symmetrically into the two $E_{8}$ factors could have smooth continuations from weak coupling to strong coupling. [77 Specifically, this $E_{7} \times$ $E_{7}$ model lies on one branch of an interesting moduli space of models. These models, in general, have a gauge group of the form $G=G_{F} \times G_{D}$, where $G_{F}$ is realized perturbatively by "fundamental" heterotic strings and $G_{D}$ is realized non-perturbatively by "dual" heterotic strings. The specific example we have here is a somewhat degenerate case, since it has $G_{F}=E_{7} \times E_{7}$, and $G_{D}=0$.

Where do these two kinds of heterotic strings come from? Non-perturbatively, the vacua we are considering correspond to $\mathrm{M}$ theory compactified on $K 3 \times S^{1} / \mathbf{Z}_{2}$. Recall that we found two different ways to make heterotic strings in M theory: 1) as a 2-brane suspended between end-of-the-world 9-branes, or (equivalently) as a 2-brane wrapped on $S^{1} / \mathbf{Z}_{2} ; 2$ ) as a 5-brane (of topology $K 3 \times S^{1}$ ) wrapped on $K 3$. The claim is that the first construction gives the "fundamental" heterotic string with its associated gauge group $G_{F}$, and the second one gives the "dual" heterotic string with its associated gauge group $G_{D}$. 
In this class of $(12,12)$ models there is an $S$ duality that interchanges the role of the two strings. This is reflected in the structure of the factorized anomaly polynomial, which has

$$
\begin{aligned}
& X_{4}=\operatorname{tr} R^{2}-\sum_{\alpha} v_{\alpha} \operatorname{tr} F_{\alpha}^{2} \\
& \tilde{X}_{4}=\operatorname{tr} R^{2}-\sum_{i} v_{i} \operatorname{tr} F_{i}^{2},
\end{aligned}
$$

where $G_{F}=\prod G_{\alpha}$ and $G_{D}=\prod G_{i}$. We will refer to these models as DMW models, since they were introduced by Duff, Minasian, and Witten. [77] The parameters $v_{\alpha}$ and $v_{i}$ take the perturbative values (listed earlier) for $G_{\alpha}$ and $G_{i}$, respectively. Thus the $G_{F}$ field strengths do not appear in $\tilde{X}_{4}$ and the $G_{D}$ field strengths do not appear in $X_{4}$. The $S$ duality transformation $\phi \rightarrow-\phi, H \rightarrow e^{-2 \phi} * H$ interchanges the Bianchi identity and the field equation for $H$. This means that it interchanges $X_{4}$ and $\tilde{X}_{4}$, and hence $G_{F}$ and $G_{D}$. Thus in one picture $G_{F}$ is realized perturbatively and $G_{D}$ is realized non-perturbatively, while after the $S$ duality transformation the situation is reversed. In this case the duality is called "heterotic string - heterotic string duality." 855 This duality is to be contrasted with the "type IIA string - heterotic string duality" discussed earlier.

By a remarkable coincidence, a dual type I construction of the same class of models was discovered independently by Gimon and Polchinski, [86] and posted to the hep-th archives on the same day as the Duff, Minasian, Witten paper. (For earlier related work see ref. [87].) The GP construction considers type I superstrings compactified on the orbifold $T^{4} / \mathbf{Z}_{2}$. This orbifold, which is a singular limit of a $K 3$, has 16 fixed points. To make a consistent model, it is necessary to arrange for the cancellation of certain tadpoles introduced by the orientifold projection used to define the type I theory. This requires the introduction of 32 Dirichlet 9-branes and 32 Dirichlet 5-branes. The 9-branes would give an $S O(32)$ gauge group in 10d, but after the compactification on $T^{4} / \mathbf{Z}_{2}$ to $6 \mathrm{~d}$ it turns out that "99 open strings" can give at most a $U(16)$ gauge group. This can be Higgsed to various subgroups. We will refer to the gauge group arising in this way as $G_{9}$. The 325 -branes are required to clump in groups of four (as in subsection 2), so they give eight dynamical 5-branes.

Recall the $S O(32)$ condition $n_{1}+n_{5}=24$. This is satisfied in the GP model by $n_{5}=8$. The reason that the model has $n_{1}=16$ is that each of the orbifold points contains a "hidden" instanton, as can be demonstrated by blowing up the singularity. 88 However, this blowup does not give the $n_{1}=16, n_{5}=8$ model described in subsection 2 . The reason for 
this is that the instantons are embedded in the $S O(32)$ group differently than they were in the examples discussed previously. The relevant embedding uses the maximal subgroup $S O(4 n) \supset S p(n) \times S U(2)$ for the case $n=8$. This accounts for the fact that an $S p(8)$ gauge group can be obtained when the 5-branes coincide away from the orbifold points. At an orbifold point it is possible to have one-half of a dynamical 5-brane (which is two Dirichlet 5-branes). This means that there are $2^{15}$ topological sectors according to which of the orbifold points have a half-integral number of 5 -branes attached. In fact, a $T$ duality transformation (on the $T^{4} / \mathbf{Z}_{2}$ ) interchanges the 5-branes and 9-branes, so there are also $2^{15}$ topological sectors for the 9-branes, giving $2^{30}$ altogether.

It turns out that there are new non-perturbative anomalies that rule out most of these topological sectors. The issue, roughly, (see Ref. [88 for details) is that these sectors would give states belonging to the wrong spin(32) conjugacy classes. Consistency allows for there to be either 0,8 , or 16 half 5-branes attached to orbifold points and similarly for the 9-branes. Thus, in view of the symmetry between them, there are altogether six topologically distinct sectors.

Let us now consider the gauge groups that can be obtained in the GP construction. As we have seen before, if $n$ dynamical 5 -branes coincide at a non-singular point of $T^{4} / \mathbf{Z}_{2}$, the 55 open strings connecting them give a $S p(n)$ gauge group. If, on the other hand, $m / 2$ dynamical 5 -branes coincide at one of the $T^{4} / \mathbf{Z}_{2}$ orbifold points, the 55 open strings connecting them turn out to give a $U(m)$ gauge group. Thus, altogether, the gauge group arising from 55 open strings is 86

$$
\begin{gathered}
G_{5}=\prod_{I} U\left(m_{I}\right) \cdot \prod_{J} S p\left(n_{J}\right) \\
\frac{1}{2} \sum m_{I}+\sum n_{J}=8 .
\end{gathered}
$$

The largest group possible - $U(16)$ - is realized if all of the 5-branes are at a single orbifold point. This satisfies the non-perturbative criterion given above. The structure of $G_{9}$, the group given by 99 open strings is exactly the same, as required by $T$ duality. Thus altogether the gauge group of a GP model is $G=G_{5} \times G_{9} \subseteq U(16) \times U(16)$. The $U(1)$ factors are broken by the mechanism described earlier, 88 so actually $G_{\max }=S U(16) \times S U(16)$.

There are various massless hyper multiplets in the spectra of 99, 59, and 55 open strings. 
Aside from $U(1)$ terms of the form $F \wedge Y_{6}$, they result in an anomaly polynomial $X_{4} \wedge \tilde{X}_{4}$ with

$$
\begin{aligned}
& X_{4}=\operatorname{tr} R^{2}-2 \sum_{\alpha} \operatorname{tr} F_{\alpha}^{2} \\
& \tilde{X}_{4}=\operatorname{tr} R^{2}-2 \sum_{i} \operatorname{tr} F_{i}^{2},
\end{aligned}
$$

where, now, $G_{5}=\prod G_{\alpha}$ and $G_{9}=\prod G_{i}$. The reason that the coefficients are all $v_{\alpha}=v_{i}=2$ is because the groups are unitary or symplectic groups, for which $v=2$ is the perturbative value. Note that in the GP construction the entire $G_{5} \times G_{9}$ is realized perturbatively, since both factors are associated with weakly coupled open strings. Moreover, the interchange of the two groups $G_{5} \leftrightarrow G_{9}$ is achieved by a $T$ duality transformation.

Clearly the DMW and GP models are closely related, but what is the exact correspondence? In the DMW picture the two groups are carried by two kinds of heterotic strings, related by an $S$ duality, whereas in the GP picture the two groups are carried by two kinds of open strings, related by a $T$ duality. Recall that in the $10 \mathrm{~d} S O(32)$ theory the group was also carried by either heterotic or open strings. In that case the heterotic string is a BPS soliton of a type I theory, and the two descriptions are $S$ dual. In the $6 \mathrm{~d}$ problem being considered now, the story is similar. Both kinds of heterotic strings, F and D, are solitons ( $D$-branes, in fact) of the type I description. 88 One corresponds to the $10 \mathrm{~d}$ heterotic string and the other corresponds to the dual 10d 5-brane wrapped on the orbifold. $T$ duality interchanges these two solitons. It is less straightforward to look for the open strings in the heterotic construction since they are not BPS solitons. Altogether, the DMW and GP models are $U$ dual, since the mapping between them turns the $S$ duality of the DMW picture into the $T$ duality of the GP picture. This is an example of "duality of dualities." These models also have dual descriptions in terms of type IIA theory 89 and F theory, 90 but since I've gone on long enough already, that will have to wait for another occasion.

\section{References}

[1] M.B. Green, J.H. Schwarz, and E. Witten, Superstring Theory in 2 vols. (Cambridge Univ. Press, 1987). 
[2] E. Cremmer, J. Scherk, and S. Ferrara, Phys. Lett. 74B (1978) 61; E. Cremmer and J. Scherk, Phys. Lett. 74B (1978) 341.

[3] E. Cremmer and B. Julia, Phys. Lett. 80B (1978) 48; Nucl. Phys. B159 (1979) 141.

[4] A. Salam and E. Sezgin, eds., Supergravities in Diverse Dimensions, reprints in 2 vols., World Scientific (1989).

[5] C. Hull and P. Townsend, "Unity of Superstring Dualities," Nucl. Phys. B438 (1995) 109, hep-th/9410167.

[6] A. Font, L. Ibañez, D. Lüst, and F. Quevedo, Phys. Lett., B249 (1990) 35; S.J. Rey, Phys. Rev. D43 (1991) 526.

[7] K. Narain, Phys. Lett. B169 (1986) 41; K. Narain, H. Sarmadi, and E. Witten, Nucl. Phys. B279 (1987) 369.

[8] A. Sen, Nucl. Phys. B404 (1993) 109, hep-th/9207053; Phys. Lett. B303 (1993) 22, hep-th/9209016; Int. J. Mod. Phys. A8 (1993) 5079, hep-th/9302038; Int. J. Mod. Phys. A8 (1993) 2023, hep-th/9303057.

[9] J.H. Schwarz, p. 503 in String Theory, Quantum Gravity, and the Unification of Fundamental Interactions (World Scientific, 1993), hep-th/9209125.

[10] J.H. Schwarz and A. Sen, Nucl. Phys. B411 (1994) 35, hep-th/9304154; Phys. Lett. B312 (1993) 105, hep-th/9305185.

[11] C. Montonen and D. Olive, Phys. Lett. B72 (1977) 117; P. Goddard, J. Nuyts, and D. Olive, Nucl. Phys. B125 (1977) 1; H. Osborne, Phys. Lett. B83 (1979) 321.

[12] A. Giveon, M. Porrati, and E. Rabinovici, Phys. Rept. 244 (1994) 77, hep-th/9401139.

[13] E. Witten and D. Olive, Phys. Lett. 78B (1978) 97; G. Gibbons and C. Hull, Phys. Lett. 109B (1982) 190.

[14] A. Sen, Int. J. Mod. Phys. A9 (1994) 3707, hep-th/9402002; J.H. Schwarz, "String Theory Symmetries," hep-th/9503127.

[15] A. Sen, Phys. Lett. B329 (1994) 217, hep-th/9402032.

[16] G. Segal and A. Selby, Commun. Math. Phys. 177 (1996) 775. 
[17] M. Porrati, Phys. Lett. B377 (1996) 67, hep-th/9505187; "How to Find H-Monopoles in Brane Dynamics," hep-th/9607082.

[18] K. Landsteiner, E. López, and D.A. Lowe, "Evidence for S-Duality in N=4 Supersymmetric Gauge Theory," hep-th/9606146.

[19] M. Bershadsky, V. Sadov, and C. Vafa, Nucl. Phys. B463 (1996) 420, hep-th/9511222.

[20] J. Dai, R.G. Leigh, and J. Polchinski, Mod. Phys. Lett. A4 (1989) 2073.

[21] M. Dine, P. Huet, and N. Seiberg, Nucl. Phys. B322 (1989) 301.

[22] P. Ginsparg, Phys. Rev. D35 (1987) 648.

[23] E. Witten, Nucl. Phys. B443 (1995) 85, hep-th/9503124.

[24] J. Polchinski and E. Witten, Nucl. Phys. B460 (1996) 525, hep-th/9510169.

[25] P.K. Townsend, Phys. Lett. B350 (1995) 184, hep-th/9501068.

[26] P. Horava and E. Witten, Nucl. Phys. B460 (1996) 506, hep-th/9510209; hepth/9603142.

[27] E. Cremmer, B. Julia, and J. Scherk, Phys. Lett. 76B (1978) 409.

[28] P.K. Townsend, "P-Brane Democracy," hep-th/9507048.

[29] M.J. Duff, R.R. Khuri, and L.X. Lu, Phys. Rep. 259 (1995) 213, hep-th/9412184.

[30] G.T. Horowitz and A. Strominger, Nucl. Phys. B360 (1991) 197.

[31] R.I. Nepomechie, Phys. Rev. D31 (1985) 1921; C. Teitelboim, Phys. Lett. 167B (1986) 69.

[32] M.J. Duff and K.S. Stelle, Phys. Lett. B253 (1991) 113.

[33] R. Güven, Phys. Lett. B276 (1992) 49.

[34] M.B. Green and J.H. Schwarz, Phys. Lett. 149B (1984) 117.

[35] A. Dabholkar, Phys. Lett. B357 (1995) 307, hep-th/9506160; C. Hull, Phys. Lett. B357 (1995) 545, hep-th/9506194.

[36] A. Strominger, Nucl. Phys. B343 (1990) 167, Erratum: B353 (1990) 565. 
[37] E. Bergshoeff, M. de Roo, M.B. Green, G. Papadopoulos, and P.K. Townsend, "Duality of Type II 7-branes and 8-branes," hep-th/9601150.

[38] M.B. Green and J.H. Schwarz, Phys. Lett. 109B (1982) 444; J.H. Schwarz, Nucl. Phys. B226 (1983) 269.

[39] C. Vafa, "Evidence for F-Theory," hep-th/9602022.

[40] A. Dabholkar, G. Gibbons, J.A. Harvey, and F. Ruiz Ruiz, Nucl. Phys. B340 (1990) 33; A. Dabholkar and J.A. Harvey, Phys. Rev. Lett. 63 (1989) 478.

[41] J.H. Schwarz, Phys. Lett. B360 (1995) 13, Erratum: Phys. Lett. B364 (1995) 252, hep-th/9508143.

[42] J. Polchinski, Phys. Rev. Lett. 75 (1995) 4724, hep-th/9510017; J. Polchinski, S. Chaudhuri, and C.V. Johnson, "Notes on D-branes," hep-th/9602052.

[43] E. Witten, Nucl. Phys. B460 (1996) 335, hep-th/9510135.

[44] P.S. Aspinwall, "Some Relationships Between Dualities in String Theory," hepth/9508154.

[45] E. Bergshoeff, E. Sezgin, and P.K. Townsend, Phys. Lett. B189 (1987) 75.

[46] M.J. Duff, P.S. Howe, T. Inami, and K.S. Stelle, Phys. Lett. B191 (1987) 70.

[47] J.H. Schwarz, Phys. Lett. B367 (1996) 97, hep-th/9510086.

[48] M.J. Duff, J.T. Liu, and R. Minasian, "Eleven-dimensional Origin of String-String Duality: a One-Loop Test," hep-th/ 9506126.

[49] G. Papadopoulos and P.K. Townsend, "Intersecting M-branes," hep-th/9603087; A.A. Tseytlin, "Harmonic Superpositions of M-branes," hep-th/9604035; J.P. Gauntlett, D.A. Kastor, and J. Traschen, "Overlapping Branes in M-Theory," hep-th/9604179.

[50] J.H. Schwarz, "M Theory Extensions of T Duality," hep-th/9601077.

[51] P.K. Townsend, K. Pilch, and P. van Nieuwenhuizen, Phys. Lett. 136B (1984) 38.

[52] S. Deser, R. Jackiw, and S. Templeton, Phys. Rev. Lett. 48 (1982) 975; Ann. Phys. 140 (1982) 372 .

[53] A. Strominger, "Open P-Branes," hep-th/9512059. 
[54] M.J. Duff and J.X. Lu, Nucl. Phys. B390 (1993) 276, hep-th/9207060.

[55] P.K. Townsend, Phys. Lett. B373 (1996) 68, hep-th/9512062.

[56] C. Callan, J.A. Harvey, and A. Strominger, Nucl. Phys. B367 (1991) 60.

[57] K. Becker and M. Becker, "Boundaries in M-Theory," hep-th/9602071.

[58] O. Aharony, J. Sonnenschein, and S. Yankielowicz, "Interactions of Strings and D-branes from M Theory," hep-th/9603009.

[59] J. Strathdee, Int. J. Mod. Phys. A2 (1987) 273.

[60] M.J. Duff, Nucl. Phys. B442 (1995) 47, hep-th/9501030.

[61] J.A. Harvey and A. Strominger, Nucl. Phys. B449 (1995) 535, Erratum: B458 (1996) 456, hep-th/9504047.

[62] P.K. Townsend, Phys. Lett. B354 (1995) 247, hep-th/9504095.

[63] A. Sen, Nucl. Phys. B450 (1995) 103, hep-th/9504027.

[64] P.S. Aspinwall, Phys. Lett. B357 (1995) 329, hep-th/9507012.

[65] J.H. Schwarz and A. Sen, Phys. Lett. B357 (1995) 323, hep-th/9507027.

[66] V.V. Nikulin, Trans. Moscow Math. Soc. 2 (1980) 71; S. Mukai, Invent. Math. 94 (1988) 183.

[67] S. Chaudhuri and D. Lowe, Nucl. Phys. B459 (1996) 113, hep-th/9508144; hepth/9512226.

[68] S. Chaudhuri and J. Polchinski, Phys. Rev. D52 (1995) 7168, hep-th/9506048.

[69] P.K. Townsend, Phys. Lett. 139B (1984) 283.

[70] K. Dasgupta and S. Mukhi, Nucl. Phys. B465 (1996) 399, hep-th/9512196.

[71] E. Witten, Nucl. Phys. B463 (1996) 383, hep-th/9512219.

[72] E. Witten, "Some Comments on String Dynamics," hep-th/9507121.

[73] E. Verlinde, Nucl. Phys. B455 (1995) 211, hep-th/9506011.

[74] M.B. Green, J.H. Schwarz, and P.C. West, Nucl. Phys. B254 (1985) 327.

[75] A. Sagnotti, Phys. Lett. 294B (1992) 196, hep-th/9210127. 
[76] E. Witten, Phys. Lett. 149B (1984) 351; M. Dine, N. Seiberg, and E. Witten, Nucl. Phys. B289 (1987) 589.

[77] M.J. Duff, R. Minasian, and E. Witten, Nucl. Phys. B465 (1996) 413, hep-th/9601036.

[78] J. Erler, J. Math. Phys. 35 (1994) 1819, hep-th/9304104.

[79] J.H. Schwarz, Phys. Lett. B371 (1996) 223, hep-th/9512053.

[80] E. Witten, Nucl. Phys. B460 (1996) 541, hep-th/9511030.

[81] M. Duff, S. Ferrara, R.R. Khuri, and J. Rahmfeld, Phys. Lett. B356 (1995) 479, hep-th/ 9506057; M. Duff, H. Lü, and C.N. Pope, "Heterotic Phase Transitions and Singularities of the Gauge Dyonic String," hep-th/9603037.

[82] O.J. Ganor and A. Hanany, "Small $E_{8}$ Instantons and Tensionless Strings," hepth/9602120.

[83] N. Seiberg and E. Witten, "Comments on String Dynamics in Six Dimensions," hep-th/ 9603003.

[84] E. Witten, "Phase Transitions in M-Theory and F-Theory," hep-th/9603150.

[85] G. Aldazabal, A. Font, L.E. Ibañez, and F. Quevedo, "Heterotic/Heterotic Duality in $\mathrm{D}=6$ and $\mathrm{D}=4$, hep-th/9602097.

[86] E.G. Gimon and J. Polchinski, "Consistency Conditions for Orientifolds and DManifolds, hep-th/9601038.

[87] G. Pradisi and A. Sagnotti, Phys. Lett. B216 (1989) 59; M. Bianchi and A. Sagnotti, Nucl. Phys. B361 (1991) 519.

[88] M. Berkooz, R.G. Leigh, J. Polchinski, J.H. Schwarz, N. Seiberg, and E. Witten, "Anomalies, Dualities, and Topology of D=6 N=1 Superstring Vacua," hep-th/9605184.

[89] P.S. Aspinwall and M. Gross, "Heterotic-Heterotic String Duality and Multiple K3 Fibrations," hep-th/9602118.

[90] D. Morrison and C. Vafa, "Compactifications of F-Theory on Calabi-Yau Three-Folds - I and II," hep-th/9602114 and hep-th/9603161; P.S. Aspinwall and M. Gross, "The SO(32) Heterotic String on a K3 Surface," hep-th/9605131. 